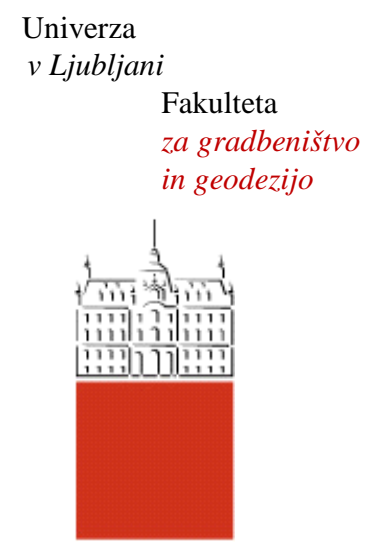

Jamova 2

1000 Ljubljana, Slovenija http://www3.fgg.uni-lj.si//

\section{DRUGG - Digitalni repozitorij UL FGG http://drugg.fgg.uni-lj.si/}

Ta članek je avtorjeva zadnja recenzirana različica, kot je bila sprejeta po opravljeni recenziji.

Prosimo, da se pri navajanju sklicujte na bibliografske podatke, kot je navedeno:
University
of Ljubljana

Faculty of

Civil and Geodetic

Engineering

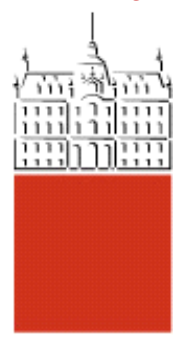

Jamova 2

SI - 1000 Ljubljana, Slovenia

http://www3.fgg.uni-lj.si/

DRUGG - The Digital Repository http://drugg.fgg.uni-lj.si/

This version of the article is author's manuscript as accepted for publishing after the review process.

When citing, please refer to the publisher's bibliographic information as follows:

Dujc, J., Brank, B., Ibrahimbegović, A. 2010. Multi-scale computational model for failure analysis of metal frames that includes softening and local buckling. Comput. methods appl. mech. eng., 199, 21-22: 1371-1385.

DOI: 10.1016/j.cma.2009.09.003 


\title{
Multi-scale computational model for failure analysis of metal frames that includes softening and local buckling
}

\author{
Jaka Dujc ${ }^{1,2}$, Boštjan Brank ${ }^{1, *}$, Adnan Ibrahimbegovic ${ }^{2}$ \\ ${ }^{1}$ University of Ljubljana, \\ Faculty of Civil and Geodetic Engineering, Ljubljana, Slovenia \\ ${ }^{2}$ Ecole Normale Supérieure de Cachan, Cachan, France \\ *corresponding author, E-mail: bbrank@fgg.uni-lj.si
}

\begin{abstract}
In this work we present a new modelling paradigm for computing the complete failure of metal frames by combining the stress-resultant beam model and the shell model. The shell model is used to compute the material parameters that are needed by an inelastic stress-resultant beam model; therefore, we consider here the shell model as the meso-scale model and the beam model as the macro-scale model. The shell model takes into account elastoplasticity with strain-hardening and strain-softening, as well as geometrical nonlinearity (including local buckling of a part of a beam). By using results of the shell model, the stress-resultant inelastic beam model is derived that takes into account elastoplasticity with hardening, as well as softening effects (of material and geometric type) in the fractureprocess zone. The beam softening effects are numerically modelled in a localized failure point by using beam finite element with embedded discontinuity. The original feature of the proposed multi-scale (i.e. shellbeam) computational model is its ability to incorporate both material and geometrical instability contributions into the stress-resultant beam model softening response. Several representative numerical simulations are presented to illustrate a very satisfying performance of the proposed approach.
\end{abstract}

\section{Introduction}

The limit load analysis and the complete failure (collapse) analysis of a structural system are important problems in performance-based design procedure. The same is true for structural dynamics. A typical example is the push-over analysis in earthquake engineering; a non-linear static analysis of a building structure, subjected to an equivalent static loading that is pushing a structure over its limit capacity (e.g. Fajfar et al. [1]).

It has been observed from failure modes, produced by seismic activities and experimental tests, that practical frame structures, composed of columns and beams, fail by exhibiting localized failures in a limited number of critical zones. Those critical zones are usually described as plastic (inelastic) hinges. A usual 
approach to compute the limit load of a structural frame, or to compute its complete failure, is to model plastic hinges with nonlinear inelastic spring finite elements. Inelastic springs are introduced at predefined critical locations in a mesh of conventional elastic beam finite elements (e.g. Wilson [2]), or, alternatively (e.g. Powell [3]), elastic beam elements with lumped nonlinear spring at both ends are used.

When studying the full collapse of a frame, a softening response is observed after reaching its limit capacity; the load reduces with additional frame deformation. This structural softening response can be modelled by an elastoplastic constitutive model with a softening relation between the generalized strain measures and the corresponding stresses resultants. However, inclusion of strain-softening in the standard finite element approximation results in a physically unrealistic mesh-dependent numerical solutions. Different alternatives have been proposed to solve this mesh-dependency (e.g. see Ibrahimbegovic [4] for a recent review). All of them are related to regularization of ill-posed mathematical problem, which arises as a result of inclusion of strain-softening in the elastoplastic constitutive model.

The most frequently used regularization nowadays is so-called embedded (strong) discontinuity approach; see e.g. Jirasek [5], Armero and Ehrlich [6], [7], [8], Ibrahimbegovic et al. [9], [10], and Wackerfuss [11] for implementation of embedded discontinuity approach for beam and bar finite elements. The key point is introduction of localized energy dissipation. This is achieved by introducing strong discontinuity in kinematic fields (e.g. a jump in rotation of the beam axis), and defining local dissipative mechanism at that discontinuity in terms of a softening cohesive law (e.g. a softening law between the bending moment and the rotation jump). Localized dissipative mechanism eliminates the mesh-dependency of numerical solutions. For beams, the introduced discontinuity can be naturally regarded as a softening plastic hinge.

In the first part of this work, we carry on with the developments related to numerical treatment of localized failure in beams in order to study failure of elastoplastic metal frames. To this end, we derive a planar straight stressresultant beam finite element with the following features: (i) Euler-Bernoulli kinematics, (ii) an elastoplastic stress-resultant constitutive model with isotropic hardening, (iii) a localized softening plastic hinge related to the strong discontinuity in generalized displacements, and (iv) an approximation of the geometrically nonlinear effects by using the von Karman strains for the virtual axial deformations.

The derived finite element can be effectively used for the limit load analysis, the push-over analysis and the complete failure analysis of planar metal frames. Localized softening, introduced by embedded discontinuity approach, solves the problem of mesh-dependency. Moreover, the spreading of plasticity over the entire frame and the appearance of the softening plastic hinges in the frame is consistently accounted for in the course of the nonlinear analysis. With respect to the existing embedded discontinuity beam finite elements, see Armero et al. [6], [7], [8], and Wackerfuss [11], we use more complex material models: stressresultant elastoplasticity with hardening to describe beam material behavior and stress-resultant rigid-plastic softening to describe material behavior at the discontinuity.

The second part of this work pertains to a procedure that provides characteristic values of material parameters, used by chosen inelastic models. Those 
values are the yield and the failure (ultimate resistance) moments of the beam cross-section, the hardening modulus for the stress-resultant beam plasticity, and the softening modulus for the softening plastic hinge. Ideally, one should for any geometry of beam cross-section, any material type and any type of beam stress state seek the appropriate experimental results and fit to them the beam model material parameters with respect to significant quantities (e.g. forces, displacements, energy, dissipation), see e.g. Kucerova et al. [12]. In the absence of experimental results for metal beams to make any definitive conclusions, we turn to another approach that belongs under multi-scale label.

The material parameters are obtained by numerical simulations on representative part of a beam by using a refined model, which is superior to the beam model in a sense that it is able to describe in more detail the beam response. We focus on rather typical metal frames with thin-walled cross-sections. For this kind of frames, the refined model can be chosen as the nonlinear shell model (e.g. Brank et al. [13], [14]). The shell model is superior to the beam model in providing a proper local description of the strain/stress fields and the overall spread of plasticity. It is also capable of describing local buckling of the flanges and the web, which is, in bending dominated conditions, very often the reason for the localized beam failure. Considering the above, the shell model can be seen as the meso-scale model and the beam model as the macro-scale model.

The outline of the paper is as follows. In section 2, we derive an elastoplastic Euler-Bernoulli beam finite element with embedded discontinuity. In section 3, we discuss computation of the beam plasticity parameters and the softening plastic hinge parameters by using the shell model. In section 4, we present details of the computational procedure. Numerical examples are presented in section 5 and concluding remarks in section 6 .

\section{Beam element with embedded discontinuity}

We consider in this section a planar Euler-Bernoulli beam finite element. The element can represent an elastoplastic bending, including the localized softening effects, which are associated with the strong discontinuity in rotation. The geometrical nonlinearity is approximately taken into account by virtual axial strains of von Karman type, which allows this element to capture the global buckling modes.

\section{$2.1 \quad$ Kinematics}

We consider a straight planar frame member, which middle axis occupies domain $\Omega \in \mathbb{R}$. Spatial discretization of $\Omega$ leads to $N_{e l}\left(\Omega=[0, L]=\cup_{e=1}^{N_{e l}} L^{(e)}\right)$ finite elements. A typical 2-node finite element is presented in Figure 1. The following notation is used: $u_{i}$ are nodal axial displacements, $w_{i}$ are nodal transverse displacements, $w_{i}^{\prime}$ are nodal values of the beam axis rotation (derivative of transverse displacement with respect to the beam axial coordinate $x \in\left[0, L^{(e)}\right]$ ), and $i=1,2$ is node number. In addition to the standard degrees of freedom at the two nodes, we assume strong discontinuity in axial displacement $\alpha_{u}$ and beam axis rotation $\alpha_{\theta}$ at $x_{d} \in L^{(e)}$. We also assume that the domain of the discontinuity influence corresponds to a single element. The axial displacement 


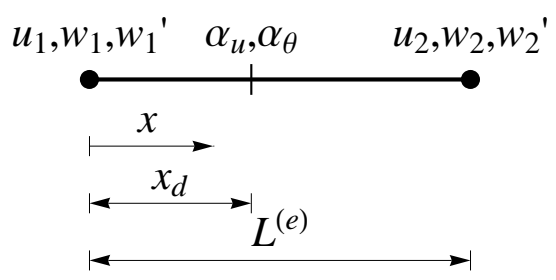

Figure 1: Beam finite element with embedded discontinuity

is thus defined as:

$$
u^{h}\left(x, x_{d}\right)=\mathbf{N}^{u}(x) \mathbf{u}+M^{u}\left(x, x_{d}\right) \alpha_{u},
$$

where $\mathbf{N}^{u}(x)=\left\{1-x / L^{(e)}, x / L^{(e)}\right\}, \mathbf{u}=\left\{u_{1}, u_{2}\right\}^{T}$, and $M^{u}\left(x, x_{d}\right)$ is a function with zero values at the nodes and a unit jump at $x_{d}$, i.e. $M^{u}\left(0, x_{d}\right)=$ $M^{u}\left(L^{(e)}, x_{d}\right)=0$ and $M^{u}\left(x_{d}^{+}, x_{d}\right)=M^{u}\left(x_{d}^{-}, x_{d}\right)+1$. Similarly, we can write the transverse displacement as

$$
w^{h}\left(x, x_{d}\right)=\mathbf{N}^{w}(x) \mathbf{w}+\mathbf{N}^{w^{\prime}}(x) \mathbf{w}^{\prime}+M^{\theta}\left(x, x_{d}\right) \alpha_{\theta},
$$

where

$$
\begin{gathered}
\mathbf{N}^{w}(x)=\left\{2\left(\frac{x}{L^{(e)}}\right)^{3}-3\left(\frac{x}{L^{(e)}}\right)^{2}+1,-2\left(\frac{x}{L^{(e)}}\right)^{3}+3\left(\frac{x}{L^{(e)}}\right)^{2}\right\}, \mathbf{w}=\left\{w_{1}, w_{2}\right\}^{T},(3) \\
\mathbf{N}^{w^{\prime}}(x)=L^{(e)}\left\{\left(\frac{x}{L^{(e)}}\right)^{3}-2\left(\frac{x}{L^{(e)}}\right)^{2}+\left(\frac{x}{L^{(e)}}\right),\left(\frac{x}{L^{(e)}}\right)^{3}-\left(\frac{x}{L^{(e)}}\right)^{2}\right\}, \mathbf{w}^{\prime}=\left\{w_{1}^{\prime}, w_{2}^{\prime}\right\}^{T},
\end{gathered}
$$

and $M^{\theta}\left(x, x_{d}\right)$ is a function with zero values at the nodes and a unit jump of its first derivative at $x_{d}$, i.e. $M^{\theta}\left(0, x_{d}\right)=M^{\theta}\left(L^{(e)}, x_{d}\right)=0$ and $M^{\theta^{\prime}}\left(x_{d}^{+}, x_{d}\right)=$ $M^{\theta^{\prime}}\left(x_{d}^{-}, x_{d}\right)+1$.

The beam axial strain can then be written as:

$$
\varepsilon\left(x, x_{d}\right)=\frac{\partial u^{h}}{\partial x}=\underbrace{\mathbf{B}^{u}(x) \mathbf{u}+G^{u}\left(x, x_{d}\right) \alpha_{u}}_{\bar{\varepsilon}}+\underbrace{\delta_{x_{d} \alpha_{u}}}_{\overline{\bar{\varepsilon}}}
$$

where $\mathbf{B}^{u}(x)=\left\{-1 / L^{(e)}, 1 / L^{(e)}\right\}, G^{u}\left(x, x_{d}\right)=\partial M^{u} / \partial x$, and $\delta_{x_{d}}$ is the Diracdelta, which appears due to discontinuous nature of axial displacement at $x_{d}$. We further divide the axial strain into a regular part $\bar{\varepsilon}$ and a singular part $\overline{\bar{\varepsilon}}$. The later can be interpreted as a localized plastic axial strain. The beam curvature is computed as:

$$
\kappa\left(x, x_{d}\right)=\frac{\partial^{2} w^{h}}{\partial x^{2}}=\underbrace{\mathbf{B}^{w}(x) \mathbf{w}+\mathbf{B}^{w^{\prime}}(x) \mathbf{w}^{\prime}+G^{\theta}\left(x, x_{d}\right) \alpha_{\theta}}_{\bar{\kappa}}+\underbrace{\delta_{x_{d}} \alpha_{\theta}}_{\overline{\bar{\kappa}}},
$$

where

$$
\begin{aligned}
\mathbf{B}^{w}(x) & =\left\{-\frac{6}{L^{(e)^{2}}}\left(1-\frac{2 x}{L^{(e)}}\right), \frac{6}{L^{(e)^{2}}}\left(1-\frac{2 x}{L^{(e)}}\right)\right\} \\
\mathbf{B}^{w^{\prime}}(x) & =\left\{-\frac{2}{L^{(e)}}\left(2-\frac{3 x}{L^{(e)}}\right),-\frac{2}{L^{(e)}}\left(1-\frac{3 x}{L^{(e)}}\right)\right\}
\end{aligned}
$$


and $G^{\theta}\left(x, x_{d}\right)=\partial^{2} M^{\theta} / \partial x^{2}$. The curvature $\kappa$ is divided into a regular part $\bar{\kappa}$ and a singular part $\overline{\bar{\kappa}}$. The later can be interpreted as a localized plastic curvature. The beam strains can be rewritten in a matrix notation as

$$
\begin{aligned}
\epsilon & =\bar{\epsilon}+\overline{\bar{\epsilon}}, \\
\bar{\epsilon} & =\underbrace{\mathbf{B d}}_{\overline{\bar{\epsilon}}}+\mathbf{G} \alpha, \quad \overline{\bar{\epsilon}}=\delta_{x_{d}} \alpha,
\end{aligned}
$$

where $\epsilon=\{\varepsilon, \kappa\}^{T}, \bar{\epsilon}=\{\bar{\varepsilon}, \bar{\kappa}\}^{T}, \widetilde{\bar{\epsilon}}=\{\widetilde{\bar{\varepsilon}}, \widetilde{\bar{\kappa}}\}^{T}, \overline{\bar{\epsilon}}=\{\overline{\bar{\varepsilon}}, \overline{\bar{\kappa}}\}^{T}$ and

$$
\begin{aligned}
& \mathbf{B}=\left\{\begin{array}{ccc}
\mathbf{B}^{u} & \mathbf{0} & \mathbf{0} \\
\mathbf{0} & \mathbf{B}^{w} & \mathbf{B}^{w^{\prime}}
\end{array}\right\}, \quad \mathbf{d}=\left\{\mathbf{u}^{T}, \mathbf{w}^{T}, \mathbf{w}^{\prime T}\right\}^{T}, \\
& \mathbf{G}=\operatorname{DIAG}\left\{G^{u}, G^{\theta}\right\}, \quad \alpha=\left\{\alpha_{u}, \alpha_{\theta}\right\}^{T} .
\end{aligned}
$$

Kinematic description of the element is concluded by derivation of $\mathbf{G}$ operator. It may be derived indirectly (i.e. without defining $M^{u}$ and $M^{\theta}$ ) through requirement that an element has to be able to describe strain-free mode at some non-zero values of $\hat{\alpha}_{u}$ and $\hat{\alpha}_{\theta}$, see Armero and Erlich [7]. According to Fig. 2,

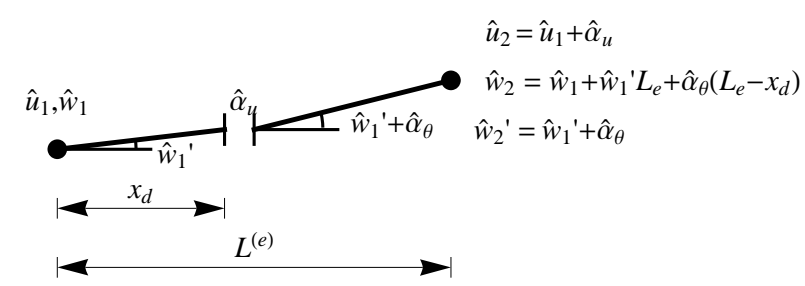

Figure 2: Strain-free mode of the element

the generalized nodal displacements $\hat{\mathbf{d}}_{\text {hinge }}=\left\{\hat{u}_{1}, \hat{u}_{2}, \hat{w}_{1}, \hat{w}_{2}, \hat{w}_{1}^{\prime}, \hat{w}_{2}^{\prime}\right\}^{T}$ of such strain-free mode are composed as

$$
\hat{\mathbf{d}}_{\text {hinge }}=\hat{\mathbf{d}}_{\text {rigid }}+\mathbf{D}_{\text {hinge }} \hat{\alpha}, \quad \mathbf{D}_{\text {hinge }}=\left[\begin{array}{cccccc}
0 & 1 & 0 & 0 & 0 & 0 \\
0 & 0 & 0 & L^{(e)}-x_{d} & 0 & 1
\end{array}\right]^{T}
$$

where $\hat{\mathbf{d}}_{\text {rigid }}=\left\{\hat{u}_{1}, \hat{u}_{1}, \hat{w}_{1}, \hat{w}_{1}+\hat{w}_{1}^{\prime} L^{(e)}, \hat{w}_{1}^{\prime}, \hat{w}_{1}^{\prime}\right\}^{T}$ are generalized nodal displacements due to rigid-body motion of a complete beam, and $\mathbf{D}_{\text {hinge }} \hat{\alpha}$ are generalized nodal displacements due to rigid-body motion of one part of the beam due to imposed strong discontinuity $\hat{\alpha}=\left\{\hat{\alpha}_{u}, \hat{\alpha}_{\theta}\right\}^{T}$. If we now set strains (9) to zero for $\hat{\mathbf{d}}_{\text {hinge }}$, we have

$$
\mathbf{0}=\mathbf{B} \hat{\mathbf{d}}_{\text {hinge }}+\mathbf{G} \hat{\alpha}=\underbrace{\mathbf{B} \hat{\mathbf{d}}_{\text {rigid }}}_{=\mathbf{0}}+\left(\mathbf{G}+\mathbf{B D}_{\text {hinge }}\right) \hat{\alpha} .
$$

Since the above equation should hold for any $\hat{\alpha}$, we get the $\mathbf{G}$ operator as

$$
\mathbf{G}=-\mathbf{B D}_{\text {hinge }}
$$

which leads to 


$$
\begin{aligned}
G^{u}\left(x, x_{d}\right) & =-\frac{1}{L^{(e)}}, \\
G^{\theta}\left(x, x_{d}\right) & =-\frac{1+3\left(1-\frac{2 x_{d}}{L^{(e)}}\right)\left(1-\frac{2 x}{L^{(e)}}\right)}{L^{(e)}} .
\end{aligned}
$$

The above definition of $\mathbf{G}$ matrix concludes kinematic description of the geometrically linear element.

Remark 1: By using (1) and (2) to describe strain-free mode of Fig. 2, one can also derive interpolation functions $M^{u}$ and $M^{\theta}$. By setting in (1) $u_{1}=\hat{u}_{1}=0, u_{2}=\hat{u}_{2}=\hat{\alpha}_{u}, u^{h}=0$ for $x<x_{d}$, and $u^{h}=0$ for $x \geq x_{d}$, one can conclude that $M^{u}=\mathrm{H}\left(x-x_{d}\right)-\mathbf{N}^{u} \cdot\{0,1\}$. Here, $\mathrm{H}\left(x-x_{d}\right)$ is unit-step function, which is 0 for $x<x_{d}$ and 1 for $x \geq x_{d}$. Derivation $\partial M^{u} / \partial x$ gives $G^{u}$ in (16). By using similar procedure for bending in (2), one can obtain $M^{\theta}=$ $\left(\mathrm{H}\left(x-x_{d}\right)\right)\left(x-x_{d}\right)-\mathbf{N}^{w} \cdot\left\{0, L^{(e)}-x_{d}\right\}-\mathbf{N}^{w^{\prime}} \cdot\{0,1\}$. Derivation $\partial^{2} M^{u} / \partial x^{2}$ gives $G^{\theta}$ in (17).

In order to account for the geometrically nonlinear effects, and related global buckling, we will use the von Karman axial strain when computing the virtual axial strain. The real axial strain, used for computing the internal forces, will still be assumed as linear, as given in eq. (5). The von Karman axial strain is defined as $\varepsilon^{V K}=\frac{\partial u^{h}}{\partial x}+\frac{1}{2}\left(\frac{\partial w^{h}}{\partial x}\right)^{2}$. The corresponding virtual axial strain is thus:

$$
\delta \varepsilon^{V K}=\frac{\partial \delta u^{h}}{\partial x}+\frac{\partial w^{h}}{\partial x} \frac{\partial \delta w^{h}}{\partial x} .
$$

If we choose to interpolate $\delta u^{h}, w^{h}$ and $\delta w^{h}$ in (18) as $\delta u^{h}=\mathbf{N}^{u}(x) \delta \mathbf{u}+$ $M^{u}\left(x, x_{d}\right) \delta \alpha_{u}, w^{h}=\mathbf{N}^{w}(x) \mathbf{w}+\mathbf{N}^{w^{\prime}}(x) \mathbf{w}^{\prime}$ and $\delta w^{h}=\mathbf{N}^{w}(x) \delta \mathbf{w}+\mathbf{N}^{w^{\prime}}(x) \delta \mathbf{w}^{\prime}$, where $\delta \mathbf{u}=\left\{\delta u_{1}, \delta u_{2}\right\}^{T}$ is vector of virtual nodal axial displacements, $\delta \mathbf{w}=$ $\left\{\delta w_{1}, \delta w_{2}\right\}^{T}$ and $\delta \mathbf{w}^{\prime}=\left\{\delta w_{1}^{\prime}, \delta w_{2}^{\prime}\right\}^{T}$ are vectors of virtual nodal transverse displacements and rotations, and $\delta \alpha_{u}$ is virtual discontinuity in axial displacement at $x_{d}$, the chosen interpolations lead to

$$
\delta \varepsilon^{V K}=\underbrace{\mathbf{B}^{u}(x) \delta \mathbf{u}+\mathbf{B}^{u, w}(x) \delta \mathbf{w}+\mathbf{B}^{u, w^{\prime}}(x) \delta \mathbf{w}^{\prime}+G^{u}\left(x, x_{d}\right) \delta \alpha_{u}}_{\delta \bar{\varepsilon}^{V K}}+\underbrace{\delta_{x_{d}} \delta \alpha_{u}}_{\delta \overline{\bar{\varepsilon}}}
$$

where

$$
\mathbf{B}^{u, w}(x)=C \frac{d \mathbf{N}^{w}}{d x}, \quad \mathbf{B}^{u, w^{\prime}}(x)=C \frac{d \mathbf{N}^{w^{\prime}}}{d x}, \quad C=\left(\frac{d \mathbf{N}^{w}}{d x} \cdot \mathbf{w}+\frac{d \mathbf{N}^{w^{\prime}}}{d x} \cdot \mathbf{w}^{\prime}\right)
$$

The linear matrix operator $\mathbf{B}$ from (11) should be thus replaced with the nonlinear matrix operator $\mathbf{B}^{V K}$ when computing virtual strains $\delta \bar{\epsilon}=\{\delta \bar{\varepsilon}, \delta \bar{\kappa}\}^{T}$, i.e.

$$
\left\{\begin{array}{c}
\delta \bar{\varepsilon}=\delta \bar{\varepsilon}^{V K} \\
\delta \bar{\kappa}
\end{array}\right\}=\underbrace{\left\{\begin{array}{ccc}
\mathbf{B}^{u} & \mathbf{B}^{u, w} & \mathbf{B}^{u, w^{\prime}} \\
\mathbf{0} & \mathbf{B}^{w} & \mathbf{B}^{w^{\prime}}
\end{array}\right\}}_{\mathbf{B}^{V K}} \delta \mathbf{d}+\mathbf{G} \delta \alpha .
$$

In (21) above, we denote with $\delta \mathbf{d}=\left\{\delta \mathbf{u}^{T}, \delta \mathbf{w}^{T}, \delta \mathbf{w}^{\prime T}\right\}^{T}$ the generalized virtual nodal displacements and with $\delta \alpha=\left\{\delta \alpha_{u}, \delta \alpha_{\theta}\right\}^{T}$ virtual jumps at $x_{d}$.

Remark 2: 
(a) The tangent stiffness matrix of the beam finite element with von Karman virtual axial strain has symmetric geometric part and non-symmetric material part. The matrix can be symmetrized by using $\mathbf{B}$ instead of $\mathbf{B}^{V K}$ when computing its material part. Such an approach would lead (for elastic beams) to the element presented in Wilson [2], section 11. In this work we use non-symmetric tangent stiffness matrix.

(b) If one uses von Karman definition of axial strains for both real and virtual strains, see Reddy [15], section 4.2, the element exhibits severe locking.

\subsection{Equilibrium equations}

The weak form of the equilibrium equations (the principle of virtual work) for an element $e$ of a chosen finite element mesh with $N_{e l}$ finite elements, can be written as:

$$
\delta \Pi^{i n t,(e)}-\delta \Pi^{e x t,(e)}=0 .
$$

By denoting the virtual strains as $\delta \epsilon=\left\{\delta \varepsilon^{V K}, \delta \kappa\right\}^{T}$, where virtual curvatures $\delta \kappa=\delta \bar{\kappa}+\delta \overline{\bar{\kappa}}$ are of the same form as real curvatures $\kappa$ in (6), we can write a single element contribution to the virtual work of internal forces as:

$$
\begin{aligned}
\delta \Pi^{i n t,(e)} & =\int_{0}^{L^{(e)}}(\delta \epsilon)^{T} \sigma d x \\
& =\underbrace{\int_{0}^{L^{(e)}} \delta \mathbf{d}^{T}\left(\mathbf{B}^{V K}\right)^{T} \sigma d x}_{\text {standard }}+\underbrace{\int_{0}^{L^{(e)}} \delta \alpha^{T}\left(\mathbf{G}^{T} \sigma+\delta_{x_{d}} \sigma\right) d x}_{\text {additional }}
\end{aligned}
$$

The matrices $\mathbf{B}^{V K}$ and $\mathbf{G}$ are defined in (21) and (15), and

$$
\sigma=\{N, M\}^{T}
$$

is the vector of beam internal forces that contains axial force $N$ and bending moment $M$. From the term "standard" in (23) we obtain the vector of element internal nodal forces

$$
\mathbf{f}^{i n t,(e)}=\int_{0}^{L^{(e)}}\left(\mathbf{B}^{V K}\right)^{T} \sigma d x .
$$

From the virtual work of external forces $\delta \Pi^{e x t,(e)}$ we can get the vector of element external nodal forces $\mathbf{f}^{e x t,(e)}$, representing the external load applied to the element. The finite element assembly of vectors $\mathbf{f}^{\text {int }},(e)$ and $\mathbf{f}^{e x t,(e)}$, for all elements of the chosen mesh, leads to a set of global (i.e. mesh related) equations

$$
A_{e=1}^{N_{e l}}\left(\mathbf{f}^{i n t,(e)}-\mathbf{f}^{e x t,(e)}\right)=\mathbf{0}
$$

where $A$ is the assembling operator.

We have only used one part of the right side of equation (23) in (22) when getting the set of global equations (26). The other term in (23), denoted as "additional" (since it results from additional enriched kinematics due to embedded discontinuity), will also contribute to the weak form of the equilibrium. However, we will treat this contribution locally element by element. Then, in 
view of (22), the following two equations are obtained for each element of the chosen mesh

$$
\begin{aligned}
\mathbf{h}^{(e)} & =\left\{h_{N}^{(e)}, h_{M}^{(e)}\right\}^{T}=\int_{0}^{L^{(e)}}\left(\mathbf{G}^{T} \sigma+\delta_{x_{d}} \sigma\right) d x \\
& =\int_{0}^{L^{(e)}} \mathbf{G}^{T} \sigma d x+\underbrace{\left.\sigma\right|_{x_{d}}}_{=\mathbf{t}}=\int_{0}^{L^{(e)}} \mathbf{G}^{T} \sigma d x+\mathbf{t}=\mathbf{0}, \quad \forall e \in\left[1, N_{e l}\right]
\end{aligned}
$$

We have defined in (27) vector $\mathbf{t}=\left.\sigma\right|_{x_{d}}=\left\{t_{N}, t_{M}\right\}^{T}$ with components $t_{N}$ and $t_{M}$ that represent axial traction and moment (bending) traction at the discontinuity. By using (17) and (24), one can obtain the component form of (27)

$$
\begin{aligned}
& h_{N}^{(e)}=\int_{0}^{L^{(e)}} G^{u} N d x+t_{N}=0, \\
& h_{M}^{(e)}=\int_{0}^{L^{(e)}} G^{\theta} M d x+t_{M}=0, \quad \forall e \in\left[1, N_{e l}\right] .
\end{aligned}
$$

The problem of solving a set of global equations (26) together with a set of local (element) equations (27) will be further addressed in section 4 .

\subsection{Constitutive relations}

We assume that the axial response of the beam material remains always elastic, thus discarding the failure by necking, for example. For the bending behavior of the beam material we choose the following constitutive models: (i) stress-resultant elastoplastic constitutive model with linear isotropic hardening, (ii) stress-resultant rigid-plasticity model with linear softening at the softening plastic hinge. The basic ingredients of the chosen constitutive relations are built on classical plasticity (e.g. Ibrahimbegovic et al. [16]) and can be summarized as:

- The regular strains $\bar{\epsilon}(10)$ can be additively decomposed into elastic part $\bar{\epsilon}^{e}$ and plastic part $\bar{\epsilon}^{p}$

$$
\bar{\epsilon}=\bar{\epsilon}^{e}+\bar{\epsilon}^{p}, \quad \bar{\epsilon}^{e}=\left\{\bar{\varepsilon}^{e}, \bar{\kappa}^{e}\right\}^{T}, \quad \bar{\epsilon}^{p}=\left\{\bar{\varepsilon}^{p}, \bar{\kappa}^{p}\right\}^{T} .
$$

- The axial strain of the beam (5) remains always elastic, thus

$$
\varepsilon=\bar{\varepsilon}=\bar{\varepsilon}^{e}, \quad \overline{\bar{\varepsilon}}=0 \quad \Longleftrightarrow \quad \bar{\varepsilon}^{p}=0, \quad \alpha_{u}=0 .
$$

- The free energy for the beam material (before localized softening is activated) is assumed to be the sum of the strain energy function $\bar{W}$ and the hardening potential $\bar{\Xi}$

$$
\bar{\Psi}\left(\bar{\epsilon}^{e}, \bar{\xi}\right):=\bar{W}\left(\bar{\epsilon}^{e}\right)+\bar{\Xi}(\bar{\xi})=\frac{1}{2} \bar{\epsilon}^{e T} \mathbf{C} \bar{\epsilon}^{e}+\frac{1}{2} K_{h} \bar{\xi}^{2},
$$

where $\mathbf{C}=D I A G\{E A, E I\}, E$ is elastic modulus, $A$ and $I$ are area and moment of inertia of cross-section, $\bar{\xi} \geq 0$ is strain-like bending hardening variable, and $K_{h} \geq 0$ is linear bending hardening modulus. 
- The yield criterion for the beam material is defined in terms of the bending moment. The admissible values of the bending moment and the stress-like bending hardening variable $\bar{q}(\bar{\xi})$ are governed by the function

$$
\bar{\phi}(M, \bar{q})=|M|-\left(M_{y}-\bar{q}\right) \leq 0,
$$

where $M_{y}>0$ denotes the positive yield moment of the cross-section. Influence of the axial force $N$ on the cross-section yielding is taken into account by defining $M_{y}$ and $\bar{q}$ as functions of $N$, as shown subsequently.

- The localization (failure) criterion that activates softening at discontinuity at $x_{d}$ is defined in terms of the bending traction $t_{M}$ and the stress-like softening bending variable $\overline{\bar{q}}(\overline{\bar{\xi}})$ (the later is defined in terms of the bending strain-like softening variable $\overline{\bar{\xi}})$

$$
\overline{\bar{\phi}}\left(t_{M}, \overline{\bar{q}}\right)=\left|t_{M}\right|-\left(M_{u}-\overline{\bar{q}}\right) \leq 0,
$$

where $M_{u}>M_{y}>0$ denotes the positive ultimate (failure) moment of the cross-section. Influence of axial force $N$ on the cross-section failure is taken into account by defining $M_{u}$ and $\overline{\bar{q}}$ as functions of $N$, as shown below.

- The bending traction $t_{M}$ at the discontinuity $x_{d}$ is related to the rotation jump as shown in Fig. 3

$$
t_{M}=t_{M}\left(\alpha_{\theta}\right)
$$

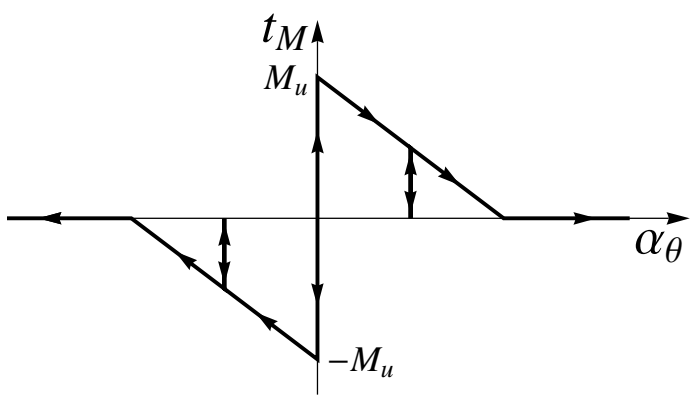

Figure 3: Rigid-plastic cohesive law at discontinuity

The remaining ingredients of the elastoplasticity with hardening can be obtained from the consideration of thermodynamics of associative plasticity and the principle of maximum plastic dissipation (see e.g. Ibrahimbegovic [4], Lubliner [17], Simo and Hughes [18]). In the present beam model the elastoplasticity with hardening happens for $\alpha=\mathbf{0}$, which leads to $\bar{\epsilon}=\widetilde{\bar{\epsilon}}$ and $\overline{\bar{\epsilon}}=\mathbf{0}$, see (10). By using (29) and (31) the mechanical dissipation can be written as

$$
0 \leq \bar{D} \stackrel{\text { def. }}{=} \sigma^{T} \dot{\bar{\epsilon}}-\dot{\bar{\Psi}}\left(\bar{\epsilon}^{e}, \bar{\xi}\right)=\left(\sigma-\frac{\partial \bar{\Psi}}{\partial \bar{\epsilon}^{e}}\right)^{T} \dot{\bar{\epsilon}}^{e}+\sigma^{T} \dot{\bar{\epsilon}}^{p}-\frac{\partial \bar{\Psi}}{\partial \bar{\xi}} \dot{\bar{\xi}}
$$


where $(\dot{o})=\partial(o) / \partial t$ and $t \in[0, T]$ is pseudo-time. By assuming that the elastic process is non-dissipative (i.e. $\bar{D}=0$ ), and that the plastic state variables do not change, we obtain from (35)

$$
\sigma=\frac{\partial \bar{\Psi}}{\partial \bar{\epsilon}^{e}}=\mathbf{C} \bar{\epsilon}^{e} \Longrightarrow N=E A \bar{\varepsilon}, M=E I\left(\bar{\kappa}-\bar{\kappa}^{p}\right) .
$$

We can define the hardening variable $\bar{q}$ by further considering (35) and (31)

$$
\bar{q}=-\frac{\partial \bar{\Psi}}{\partial \bar{\xi}}=-\frac{\partial \bar{\Xi}}{\partial \bar{\xi}}=-K_{h} \bar{\xi} .
$$

By replacing (36) and (37) in (35), the plastic dissipation can be obtained as

$$
\bar{D}^{p}=\sigma^{T} \dot{\bar{\epsilon}}^{p}+\bar{q} \dot{\bar{\xi}}^{(24),} \stackrel{(29),}{\Longrightarrow}{ }^{(30)} \bar{D}^{p}=M \dot{\bar{\kappa}}^{p}+\overline{\bar{q}} \dot{\bar{\xi}} .
$$

The principle of maximum plastic dissipation states that among all the variables $(M, \bar{q})$ that satisfy the yield criteria $\bar{\phi}(M, \bar{q}) \leq 0$, one should choose those that maximize plastic dissipation (at frozen rates $\dot{\bar{\kappa}}^{p}$ and $\dot{\bar{\xi}}$ ). This can be written as a constrained optimization problem:

$$
\min _{M, \bar{q}} \max _{\dot{\bar{\gamma}}}\left[\bar{L}^{p}(M, \bar{q}, \dot{\bar{\gamma}})=-\bar{D}^{p}(M, \bar{q})+\dot{\bar{\gamma}} \bar{\phi}(M, \bar{q})\right],
$$

where $\dot{\bar{\gamma}} \geq 0$ plays the role of Lagrange multiplier. By using (38) and (32), the last result can provide the evolution equations for internal variables

$$
\begin{aligned}
\frac{\partial \bar{L}^{p}}{\partial M}=-\dot{\bar{\kappa}}^{p}+\dot{\bar{\gamma}} \frac{\partial \bar{\phi}}{\partial M}=0 & \Longrightarrow \quad \dot{\bar{\kappa}}^{p}=\operatorname{sign}(M) \dot{\bar{\gamma}}, \\
\frac{\partial \bar{L}^{p}}{\partial \bar{q}}=-\dot{\bar{\xi}}+\dot{\bar{\gamma}} \frac{\partial \bar{\phi}}{\partial \bar{q}}=0 & \Longrightarrow \quad \dot{\bar{\xi}}=\dot{\bar{\gamma}},
\end{aligned}
$$

along with the Kuhn-Tucker loading/unloading conditions and the consistency condition

$$
\dot{\bar{\gamma}} \geq 0, \quad \bar{\phi} \leq 0, \quad \dot{\bar{\gamma}} \bar{\phi}=0, \quad \dot{\bar{\gamma}} \dot{\bar{\phi}}=0 .
$$

To obtain the remaining ingredients of the rigid-plastic response describing softening at the discontinuity $x_{d}$, let us isolate the softening plastic hinge. We first define (bending) softening potential at the discontinuity as $\overline{\bar{\Xi}}=\overline{\bar{\Psi}}=\frac{1}{2} K_{s}^{2} \overline{\bar{\xi}}$, where $\overline{\bar{\Psi}}$ is the strain energy function due to softening. The softening potential depends on the strain-like (bending) softening variable $\overline{\bar{\xi}} \geq 0$ and the linear (bending) softening modulus $K_{s} \leq 0$. The dissipation at $x_{d}$ can be then written as:

$$
0 \leq \overline{\bar{D}} \stackrel{\text { def. }}{=} t_{M} \dot{\alpha}_{\theta}-\dot{\bar{\Psi}}(\overline{\bar{\xi}})=t_{M} \dot{\alpha}_{\theta}-\frac{\partial \overline{\bar{\Psi}}}{\partial \overline{\bar{\xi}}} \dot{\bar{\xi}}
$$

where $t_{M}$ is the discontinuity bending traction given by (34). By defining

$$
\overline{\bar{q}}=-\frac{\partial \overline{\bar{\Psi}}}{\partial \overline{\bar{\xi}}}=-\frac{\partial \overline{\bar{\Xi}}}{\partial \overline{\bar{\xi}}}=-K_{s} \overline{\bar{\xi}}=\left|K_{s}\right| \overline{\bar{\xi}},
$$


the result in (43) can be rewritten as

$$
\overline{\bar{D}}=\overline{\bar{D}}^{p}=t_{M} \dot{\alpha}_{\theta}+\overline{\bar{q}} \dot{\bar{\xi}}
$$

The principle of maximum plastic dissipation at the rigid-plastic discontinuity can then be defined as:

$$
\min _{t_{M}, \overline{\bar{q}}} \max _{\overline{\bar{\gamma}}}\left[\overline{\bar{L}}^{p}\left(t_{M}, \overline{\bar{q}}, \dot{\bar{\gamma}}\right)=-\overline{\bar{D}}^{p}\left(t_{M}, \overline{\bar{q}}\right)+\dot{\bar{\gamma}} \overline{\bar{\phi}}\left(t_{M}, \overline{\bar{q}}\right)\right]
$$

where $\dot{\overline{\bar{\gamma}}} \geq 0$ is the Lagrange multiplier. By using (45) and (33), we get from (46) above the following evolution equations:

$$
\begin{aligned}
& \frac{\partial \overline{\bar{L}}^{p}}{\partial t_{M}}=-\dot{\alpha}_{\theta}+\dot{\overline{\bar{\gamma}}} \frac{\partial \overline{\bar{\phi}}}{\partial t_{M}}=0 \Longrightarrow \dot{\alpha}_{\theta}=\operatorname{sign}\left(t_{M}\right) \dot{\overline{\bar{\gamma}}} \\
& \frac{\partial \overline{\bar{L}}^{p}}{\partial \overline{\bar{q}}}=-\dot{\overline{\bar{\xi}}}+\dot{\overline{\bar{\gamma}}} \frac{\partial \overline{\bar{\phi}}}{\partial \overline{\bar{q}}}=0 \Longrightarrow \dot{\overline{\bar{\xi}}}=\dot{\overline{\bar{\gamma}}}
\end{aligned}
$$

By observing that $\operatorname{sign}\left(t_{M}\right)=\operatorname{sign}\left(\alpha_{\theta}\right)$ (see (34) and Fig. 3), it follows from (47) that

$$
\operatorname{sign}\left(\alpha_{\theta}\right) \dot{\alpha}_{\theta}=\dot{\overline{\bar{\xi}}} \Rightarrow\left|\alpha_{\theta}\right|=\overline{\bar{\xi}}
$$

The Kuhn-Tucker loading/unloading conditions and the consistency condition also apply:

$$
\dot{\overline{\bar{\gamma}}} \geq 0, \quad \overline{\bar{\phi}} \leq 0, \quad \dot{\bar{\gamma}} \overline{\bar{\phi}}=0, \quad \dot{\overline{\bar{\gamma}}} \dot{\overline{\bar{\phi}}}=0 .
$$

With the above results, we are in position to write the total dissipation of the beam finite element when the element is in the softening regime. Namely, by accounting for the proper definition of strain energy terms for the beam finite element according to $\Psi=\int_{0}^{L^{(e)}} \bar{\Psi} d x+\overline{\bar{\Psi}}$, the total dissipation in the softening regime can be written as

$$
\begin{aligned}
& \overline{\bar{D}}_{L^{(e)}}^{t o t}=\int_{0}^{L^{(e)}}\left(\sigma^{T} \dot{\bar{\epsilon}}-\dot{\bar{\Psi}}\left(\bar{\epsilon}^{e}, \bar{\xi}\right)\right) d x+\left(t_{M} \dot{\alpha}_{\theta}-\dot{\bar{\Psi}}(\overline{\bar{\xi}})\right) \\
& =\int_{0}^{L^{(e)}}(\sigma^{T} \dot{\overline{\bar{\epsilon}}}+\sigma^{T} \mathbf{G} \dot{\alpha}-\sigma^{T} \underbrace{\dot{\bar{\epsilon}}^{e}}_{\dot{\tilde{\bar{\epsilon}}}^{e}}+\overline{\bar{q}}) d x+\left(t_{M} \dot{\bar{\alpha}}_{\theta}+\overline{\bar{q}} \dot{\overline{\bar{\xi}}}\right) \\
& =\int_{0}^{L^{(e)}} \underbrace{\left(M \dot{\bar{\kappa}}^{p}+\overline{\bar{\xi}}\right)}_{\bar{D}^{p}, \text { see }(38)} d x+\underbrace{\left(\int_{0}^{L^{(e)}} G^{\theta} M d x+t_{M}\right)}_{=0, \text { see }(28)} \dot{\alpha}_{\theta}+\underbrace{\overline{\bar{q}}}_{\left|K_{s}\right| \overline{\bar{\xi}}} \dot{\overline{\bar{\xi}}}
\end{aligned}
$$

It can be seen from (51) that enforcing eq. (28) will decuple dissipation in the softening plastic hinge from the dissipation in the rest of the beam. Therefore, eq. (28) is further used to compute $t_{M}$.

We conclude description of constitutive relations by defining plastic work of the beam cross-section in the hardening regime

$$
\bar{W}^{p}=M \dot{\bar{\kappa}}^{p}=|M| \dot{\bar{\xi}}=\left(M_{y}+K_{h} \bar{\xi}\right) \dot{\bar{\xi}}
$$

and plastic work for the beam finite element in the softening regime as

$$
\overline{\bar{W}}^{p}=t_{M} \dot{\alpha_{\theta}}=\left|t_{M}\right| \dot{\bar{\xi}}=\left(M_{u}+K_{s} \overline{\bar{\xi}}\right) \dot{\bar{\xi}}
$$




\section{Computation of beam plasticity material pa- rameters}

In the previous section, we have built the framework for stress-resultant plasticity for beam finite element with embedded discontinuity. The material parameters that need to be known for chosen material models are: (i) $M_{y}$ and $K_{h}$ for the plasticity with hardening, and (ii) $M_{u}$ and $K_{s}$ for the softening plastic hinge. In this section we will elaborate on determination of the above parameters.

The yield moment $M_{y}$ can be determined by considering the uniaxial yield stress of the material $\sigma_{y}$, the bending resistance modulus of cross-section $W$, the cross-section area $A$, and the level of axial force $N$. One can associate the yield moment of the cross-section with the yielding of the most-stressed material fiber to get

$$
M_{y}(N)=W \sigma_{y}\left(1-\frac{|N|}{A \sigma_{y}}\right) .
$$

The ultimate bending moment $M_{u}$ can be derived in a closed form by assuming elastic-perfectly-plastic response of material fibers, e.g. Lubliner [17]. However, one may try to determine a better estimate for $M_{u}$, which takes into consideration material hardening, as well as possibility of local buckling (e.g. buckling of the flanges and/or the web of the I-beam). This task is addressed in the present work by performing computations with refined finite element model based on geometrically and materially nonlinear shell element, which is able to capture local buckling and gradual spreading of plasticity over the cross-section. The ultimate bending resistance $M_{u}$ can be obtained by using results of such a shell model computations, as can be moduli $K_{h}$ and $K_{s}$.

To obtain desired results, a part of the frame member with a reference length $L^{r e f}<L^{t o t}$ ( $L^{t o t}$ is the total length of the frame member under consideration) is: (i) modelled with shell finite elements, (ii) subjected to an external axial force $\widehat{N}$ in the first loading step, and (iii) subjected to a varying external bending moment at the end cross-sections in the second loading step, while keeping $\widehat{N}$ fixed, see Fig. 4 (b). It is assumed that such a loading pattern would produce approximately constant internal axial force $N=\widehat{N}$ during the analysis. The computation with shell model takes into account geometrical and material nonlinearity that include: plasticity with hardening and strain-softening, strainsoftening regularization, and local buckling effects. The results of shell analysis are cast in terms of diagrams presented in Figs. 4 (d) and (g). One can associate the ultimate bending moment $M_{u}$ with the peak point in the diagram at Fig. 4 (d), where applied end moment is plotted versus the end rotation, i.e.

$$
M_{u}(N)=M_{u}^{r e f}(N) .
$$

One can also use this point as a border-point between the hardening regime and the softening regime, where the softening can be due to material and/or geometric effects. To determine the values of the beam model hardening and softening parameters, we make an assumption that the plastic work at failure should be equal for both the beam and the shell model. In other words, we want the internal forces of the beam model to produce the same amount of the plastic work as the internal forces of the shell model, when considering the full failure of the part of the frame member of length $L^{\text {ref }}$. 

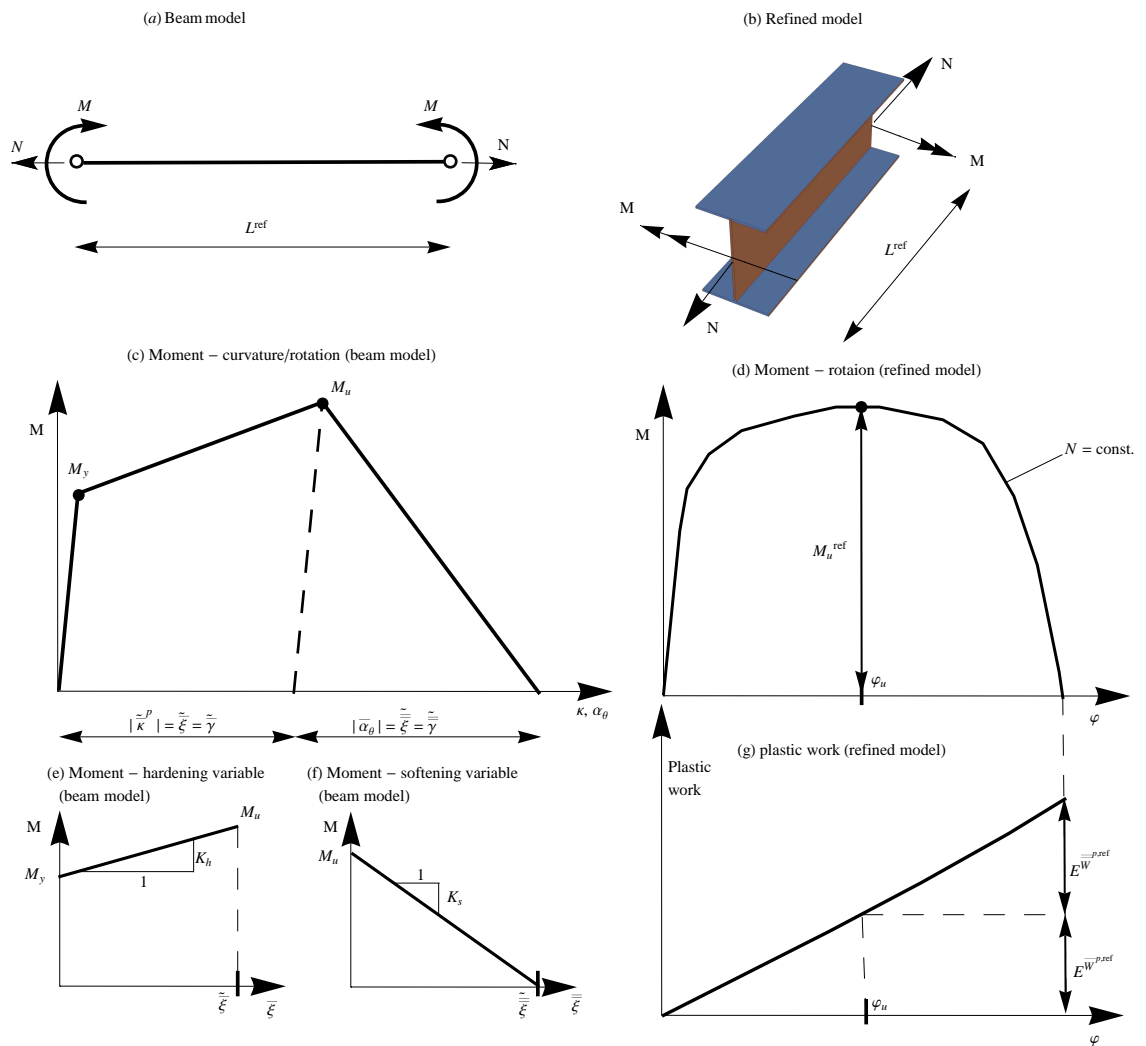

Figure 4: Evaluation of beam material parameters by using results of refined analysis

Since the plastic work is done in two regimes (hardening and softening), we have to assure that the amount of plastic work in each regime matches for both models, i.e.

$$
E^{\bar{W}^{p}}(N)=E^{\bar{W}^{p, r e f}}(N), \quad E^{\overline{\bar{W}}^{p}}(N)=E^{\overline{\bar{W}}^{p, r e f}}(N) .
$$

The plastic work in the hardening regime, $E^{\bar{W}^{p, r e f}}$, and the plastic work in the softening regime, $E^{\overline{\bar{W}}^{p, r e f}}$, are obtained from the shell model analysis, Fig. 4 (g).

The plastic work of the beam model in the hardening regime, $E^{\bar{W}^{p}}$, can be determined by observing that each cross-section in the frame member of length $L^{r e f}$ is approximately under the same force-moment state during the hardening regime. Integration of (52) allows us to write

$$
E^{\bar{W}^{p}}=\int_{0}^{L^{r e f}} \int_{0}^{t \text { at } M_{u}} \bar{W}^{p} d \tau d l=L^{r e f}\left(M_{y} \widetilde{\bar{\xi}}+\frac{1}{2} K_{h} \widetilde{\bar{\xi}}^{2}\right) .
$$


In (57) above $\widetilde{\bar{\xi}}$ is the value of hardening variable that corresponds to the bending moment $M_{u}$, Fig. 4 (e). Since we have assumed linear hardening in the beam model (37), we get

$$
\widetilde{\bar{\xi}}=\frac{M_{u}-M_{y}}{K_{h}} .
$$

By using (58), (57) and (56), one can obtain an expression for hardening modulus as

$$
K_{h}(N)=\frac{\left(M_{u}^{2}(N)-M_{y}^{2}(N)\right) L^{r e f}}{2 E^{\bar{W}^{p, r e f}}(N)} .
$$

The plastic work of beam model in the softening regime, $E^{\overline{\bar{W}}^{p}}$, can be determined by assuming that the softening part of $M-\varphi$ curve in Fig. 4 (d), obtained from the shell model analysis, is produced by a very localized phenomenon (in a single cross-section) related to the local buckling and/or to the localized strain-softening. By using (53), one can compute the plastic work in the softening regime for the beam model as

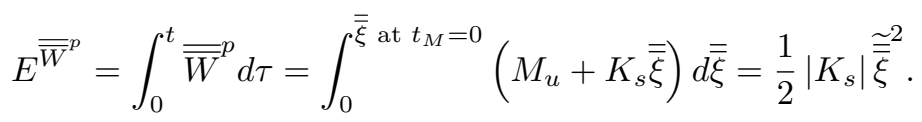

In $(60) \widetilde{\overline{\bar{\xi}}}$ is the value of the softening variable that corresponds to the total cross-section failure, Fig. 4 (f). Since we have assumed linear softening in the beam model, we obtain

$$
\stackrel{\overline{\bar{\xi}}}{=} \frac{M_{u}}{\left|K_{s}\right|} .
$$

By using (61), (60) and (56), one can obtain an expression for softening modulus as

$$
\left|K_{s}(N)\right|=\frac{M_{u}^{2}(N)}{2 E^{\overline{\bar{W}}^{p, r e f}}(N)}, \quad K_{s} \leq 0 .
$$

We note, that the choice of the reference length $L^{\text {ref }}$ should have very small influence on the values of the searched material parameters. The influence on the value of $K_{h}$ should be small, since each cross-section is approximately under the same force-moment state during the hardening regime. The influence on the value of $K_{s}$ should not be too big neither, since the softening effect is localized. However, one should perform large displacement correction of $M_{u}$, if the chosen length of $L^{\text {ref }}$ enables large deflections, as shown in example 5.4.

\section{Computational procedure}

In this section we will present a procedure for solving the set of global (mesh related) and the set of local (element related) nonlinear equations generated by using the stress-resultant plasticity beam finite element with embedded discontinuity presented in section 2 .

The solution of the set of global nonlinear equations (26), along with the set of local nonlinear equations (27) (note that (27) is reduced to (28) due to assumption (30)), ought to be computed at discrete pseudo-time values $0, t_{1}, t_{2}, \ldots, t_{n-1}, t_{n}, t_{n+1}, \ldots, T$ by means of the incremental-iterative scheme. 
We will consider the solution in a typical pseudo-time incremental step from $t_{n}$ to $t_{n+1}$. Let us assume that all the variables, related to an element $e$ and its integration points $i p=1,2,3$ (a 3-point Lobatto integration scheme is used) are given at $t_{n}$, i.e.

$$
\text { given: } \mathbf{d}_{n}^{(e)}, \bar{\kappa}_{n}^{p, i p}, \bar{\xi}_{n}^{i p}, \alpha_{\theta, n}^{(e)}, \overline{\bar{\xi}}_{n}^{(e)} \text { and } M_{y}^{i p}, x_{d}^{(e)}, M_{u}^{(e)} .
$$

We have also added in (63): (i) the yield moment at integration point $M_{y}^{i p}$ (which is only true if hardening plasticity has been activated so far) and (ii) position of the discontinuity $x_{d}^{(e)}$ and the ultimate bending moment $M_{u}^{(e)}$ (which is only true if softening plastic hinge has been activated so far). We will then iterate in the pseudo-time step in order to compute the converged values of the variables at $t_{n+1}$, i.e.

find: $\mathbf{d}_{n+1}^{(e)}, \bar{\kappa}_{n+1}^{p, i p}, \bar{\xi}_{n+1}^{i p}, \alpha_{\theta, n+1}^{(e)}, \overline{\bar{\xi}}_{n+1}^{(e)}$ and (if not given already) $M_{y}^{i p}, x_{d}^{(e)}, M_{u}^{(e)}$.

The moments $M_{y}^{i p}$ and $M_{u}^{(e)}$ are computed by using (54) and (55). Although they depend on axial force $N$, we keep them fixed once determined.

The computation of solution (64) is split into two phases:

(a) The global (mesh related) phase computes the current iterative values (with (i) as the iteration counter) of nodal generalized displacements at $t_{n+1}$ while keeping the other variables fixed, i.e.

$$
\text { global phase: } \mathbf{d}_{n+1}^{(e),(i)}=\mathbf{d}_{n+1}^{(e),(i-1)}+\Delta \mathbf{d}_{n+1}^{(e),(i-1)} .
$$

The computation of iterative update $\Delta \mathbf{d}_{n+1}^{(e),(i-1)}$ will be explained below.

(b) The local (element and integration point related) phase computes the values of variables $\bar{\kappa}_{n+1}^{p, i p}, \bar{\xi}_{n}^{i p}, \alpha_{\theta, n+1}^{(e)}, \overline{\bar{\xi}}_{n+1}^{(e)}$ while keeping $\mathbf{d}_{n+1}^{(e),(i)}$ fixed. The computation procedure depends on weather the softening plastic hinge has been activated in the considered element or not. Therefore, the local computation procedure on the level of a single element can be based either on hardening plasticity procedure or on softening plasticity procedure (excluding each other).

In the rest of this section we will first describe the local phase, which will be followed by the description of the global phase. The hardening plasticity procedure is carried out at each integration point ip (e.g. Dujc and Brank [19]). We first provide the trial value of the bending moment

$$
M_{n+1}^{\text {trial }, i p}=E I\left(\bar{\kappa}\left(\mathbf{d}_{n+1}^{(e),(i)}, \alpha_{\theta, n}^{(e)}\right)-\bar{\kappa}_{n}^{p, i p}\right),
$$

and the trial value of the yield function $\bar{\phi}^{\text {trial,ip }}$. If the trial yield criterion

$$
\bar{\phi}^{\text {trial }, i p}\left(M_{n+1}^{\text {trial }, i p}, \bar{q}\left(\bar{\xi}_{n}^{i p}\right)\right) \stackrel{?}{\leq} 0
$$

is satisfied, the values of hardening plasticity local variables remain unchanged (the step is elastic)

$$
\bar{\phi}^{\text {trial }, i p} \leq 0 \quad \Longrightarrow \quad \bar{\kappa}_{n+1}^{p, i p}=\bar{\kappa}_{n}^{p, i p}, \quad \bar{\xi}_{n+1}^{i p}=\bar{\xi}_{n}^{i p} .
$$


In the case of violation of the trial yield criterion (67), the values of local variables are updated by backward Euler integration scheme

$$
\bar{\kappa}_{n+1}^{p, i p}=\bar{\kappa}_{n}^{p, i p}+\operatorname{sign}\left(M_{n+1}^{\text {trial }, i p}\right) \bar{\gamma}_{n+1}^{i p}, \quad \bar{\xi}_{n+1}^{i p}=\bar{\xi}_{n}^{i p}+\bar{\gamma}_{n+1}^{i p},
$$

where $\bar{\gamma}_{n+1}^{i p}=\dot{\bar{\gamma}}_{n+1}^{i p}\left(t_{n+1}-t_{n}\right)$. The value of the plastic multiplier $\bar{\gamma}_{n+1}^{i p}$ is determined from

$$
\bar{\phi}^{i p}\left(M_{n+1}^{i p}\left(\mathbf{d}_{n+1}^{(e),(i)}, \bar{\kappa}_{n+1}^{p, i p}\left(\bar{\gamma}_{n+1}^{i p}\right)\right), \bar{q}\left(\bar{\xi}_{n+1}^{i p}\left(\bar{\gamma}_{n+1}^{i p}\right)\right)\right)=\bar{\phi}^{i p}\left(\bar{\gamma}_{n+1}^{i p}\right)=0 .
$$

For the linear hardening one can determine $\bar{\gamma}_{n+1}^{i p}$ explicitly. For a nonlinear hardening an iteration procedure has to be used. The main result of the above described hardening plasticity procedure is the new values of the bending moment $M_{n+1}^{i p}$, computed as

$$
M_{n+1}^{i p}=E I\left(\bar{\kappa}\left(\mathbf{d}_{n+1}^{(e),(i)}, \alpha_{\theta, n}^{(e)}\right)-\bar{\kappa}_{n+1}^{p, i p}\right),
$$

and the elastoplastic tangent operator $\partial M_{n+1}^{i p} / \partial \kappa_{n+1}^{i p,(i)}$. The updated value of the rotation jump is $\alpha_{\theta, n+1}^{(e)}=\alpha_{\theta, n}^{(e)}$.

The softening plasticity procedure is carried out at each finite element $e$. The discontinuity $x_{d}^{(e)}$ can only appear at the position of the integration point with the largest absolute value of the bending moment. We first provide the trial value of the bending traction at the discontinuity

$$
t_{M, n+1}^{\text {trial, (e) }}=-\int_{0}^{L^{(e)}} G^{\theta}\left(x_{d}^{(e)}, x\right) M\left(\mathbf{d}_{n+1}^{(e),(i)}, \bar{\kappa}_{n}^{p, i p}, \alpha_{\theta, n}^{(e)}\right) d x .
$$

and the trial value of the failure function

$$
\overline{\bar{\phi}}^{\text {trial },(e)}\left(t_{M, n+1}^{\text {trial },(e)}, \overline{\bar{q}}\left(\overline{\bar{\xi}}_{n}^{(e)}\right)\right) \stackrel{?}{\leq} 0 .
$$

If the trial failure criterion (73) is satisfied, the values of softening plasticity local variables remain unchanged

$$
\overline{\bar{\phi}}^{\text {trial },(e)} \leq 0 \quad \Longrightarrow \quad \alpha_{\theta, n+1}^{(e)}=\alpha_{\theta, n}^{(e)}, \quad \overline{\bar{\xi}}_{n+1}^{(e)}=\overline{\bar{\xi}}_{n}^{(e)} .
$$

In the case of violation of the trial yield criterion (73), the values of local variables are updated by backward Euler integration scheme

$$
\alpha_{\theta, n+1}^{(e)}=\alpha_{\theta, n}^{(e)}+\operatorname{sign}\left(t_{M, n+1}^{t r i a l,(e)}\right) \overline{\bar{\gamma}}_{n+1}^{(e)}, \quad \overline{\bar{\xi}}_{n+1}^{(e)}=\overline{\bar{\xi}}_{n}^{(e)}+\overline{\bar{\gamma}}_{n+1}^{(e)},
$$

where $\overline{\bar{\gamma}}_{n+1}^{(e)}=\dot{\bar{\gamma}}_{n+1}^{(e)}\left(t_{n+1}-t_{n}\right)$. The value of the plastic multiplier $\overline{\bar{\gamma}}_{n+1}^{(e)}$ is determined from condition

$$
\overline{\bar{\phi}}^{(e)}\left(t_{M, n+1}^{(e)}\left(\alpha_{\theta, n+1}^{(e)}\left(\overline{\bar{\gamma}}_{n+1}^{(e)}\right)\right), \overline{\bar{q}}\left(\overline{\bar{\xi}}_{n+1}^{(e)}\left(\overline{\bar{\gamma}}_{n+1}^{(e)}\right)\right)\right)=\overline{\bar{\phi}}^{(e)}\left(\overline{\bar{\gamma}}_{n+1}^{(e)}\right)=0 .
$$

For the linear softening one can determine the plastic multiplier explicitly, whereas for nonlinear softening an iterative solution procedure is needed. Note, that we compute the bending traction in (76) as

$$
t_{M, n+1}^{(e)}=-\int_{\Omega^{e}} G^{\theta}\left(x_{d}^{(e)}, x\right) M\left(\mathbf{d}_{n+1}^{(e),(i)}, \bar{\kappa}_{n}^{p, i p}, \alpha_{\theta, n+1}^{(e)}\right) d x .
$$


The main result of the above described softening plasticity procedure is the new value of softening variable $\alpha_{\theta, n+1}^{(e)}$, which influences the stress state of the whole element by giving the new values of the bending moment $M_{n+1}^{i p}$ as

$$
M_{n+1}^{i p}=E I\left(\bar{\kappa}\left(\mathbf{d}_{n+1}^{(e),(i)}, \alpha_{\theta, n+1}^{(e)}\right)-\bar{\kappa}_{n}^{p, i p}\right) .
$$

The updated value of the plastic curvature is $\bar{\kappa}_{n+1}^{p, i p}=\bar{\kappa}_{n}^{p, i p}$.

Once the local variables are computed, we turn to the global phase of the iterative loop in order to provide, if so needed, new iterative values of nodal displacements. First, the set of global equilibrium equations (26) is checked with newly computed $M_{n+1}^{i p}$ from the local phase

$$
\left\|A_{e=1}^{N_{e l}}\left[\mathbf{f}_{n+1}^{i n t,(e)}-\mathbf{f}_{n+1}^{e x t,(e),(i)}\right]\right\| \stackrel{?}{<} t o l .
$$

If the convergence criterion (79) is satisfied, we move on to the next pseudo-time incremental step. If the convergence criterion fails, we perform a new iterative sweep within the present pseudo-time incremental step. New iterative values of nodal generalized displacements of the finite element mesh are computed by accounting for each element contribution. A single element contribution can be written as

$$
\left[\begin{array}{ll}
\mathbf{K}^{(e)} & \mathbf{K}^{f \alpha} \\
\mathbf{K}^{h d} & K^{h \alpha}
\end{array}\right]_{n+1}^{(i)}\left(\begin{array}{c}
\Delta \mathbf{d}_{n+1}^{(e),(i)} \\
\Delta \alpha_{\theta, n+1}^{(e),(i)}
\end{array}\right)=\left(\begin{array}{c}
\mathbf{f}_{n+1}^{e x t,(e)}-\mathbf{f}_{n+1}^{i n t,(e),(i)} \\
0
\end{array}\right)
$$

where the parts of the element stiffness matrix can be formally written as

$$
\begin{array}{ll}
\mathbf{K}_{n+1}^{(e),(i)}=\left(\frac{\partial \mathbf{f}^{i n t,(e)}}{\partial \mathbf{d}^{(e)}}\right)_{n+1}^{(i)}, & \mathbf{K}_{n+1}^{f \alpha,(i)}=\left(\frac{\partial \mathbf{f}^{i n t,(e)}}{\partial \alpha_{\theta}^{(e)}}\right)_{n+1}^{(i)}, \\
\mathbf{K}_{n+1}^{h d,(i)}=\left(\frac{\partial h_{M}^{(e)}}{\partial \mathbf{d}^{(e)}}\right)_{n+1}^{(i)}, & K_{n+1}^{h \alpha,(i)}=\left(\frac{\partial h_{M}^{(e)}}{\partial \alpha_{\theta}^{(e)}}\right)_{n+1}^{(i)}
\end{array}
$$

The static condensation of (80) allows us to form the element stiffness matrix $\widehat{\mathbf{K}}_{n+1}^{(e),(i)}$ that contributes to the assembly

$$
\left.A_{e=1}^{N_{e l} l}\left(\widehat{\mathbf{K}}_{n+1}^{(e),(i)}\right] \Delta \mathbf{d}_{n+1}^{(i)}\right)=A_{e=1}^{N_{e l}}\left(\mathbf{f}_{n+1}^{e x t,(e)}-\mathbf{f}_{n+1}^{i n t,(e),(i)}\right),
$$

where

$$
\widehat{\mathbf{K}}_{n+1}^{(e),(i)}=\mathbf{K}_{n+1}^{(e),(i)}-\mathbf{K}_{n+1}^{f \alpha,(i)}\left(K_{n+1}^{h \alpha,(i)}\right)^{-1} \mathbf{K}_{n+1}^{h d,(i)} .
$$

Solution of (82) gives the values of iterative update $\Delta \mathbf{d}_{n+1}^{(e),(i)}$, which leads us back to $(65)$.

\section{$5 \quad$ Examples}

In this section we illustrate performance of the above derived beam element when analyzing push-over and collapse of steel frames. We also illustrate the procedure, presented in section 3 , for computing the beam model plasticity material parameters by using the shell finite element model. The beam model computer code was generated by using symbolic manipulation code AceGen and the examples were computed by using finite element program AceFem, see Korelc [20]. 


\subsection{Computation of beam plasticity material parameters}

With this example we illustrate computation of beam plasticity material parameters $M_{u}, K_{h}$ and $K_{s}$ as suggested in section 3 . We consider a frame member with an I-cross-section with flange width $b_{f}=30 \mathrm{~cm}$, flange thickness $t_{f}=1.5$ $\mathrm{cm}$, web height $b_{w}=40 \mathrm{~cm}$ and web thickness $t_{w}=0.8 \mathrm{~cm}$. The cross-section area is $A=122 \mathrm{~cm}^{2}$ and the bending resistance modulus is $I=43034.2 \mathrm{~cm}^{4}$. We model a part of the frame member of length $L^{r e f}=2 L=300 \mathrm{~cm}$, which is 7 times the height of the section. This length should be sufficient to capture the local softening effects due to local buckling and/or strain softening. The frame member is made of an elastoplastic material (steel), whose uniaxial response is plotted in Fig. 5. Young's modulus is $E=21000 \mathrm{kN} / \mathrm{cm}^{2}$, yield stress is $\sigma_{y}=24 \mathrm{kN} / \mathrm{cm}^{2}$, ultimate stress is $\sigma_{u}=36 \mathrm{kN} / \mathrm{cm}^{2}$, yield strain is $\varepsilon_{y}=\sigma_{y} / E$, strain at ultimate stress is $\varepsilon_{u}=0.1$ and strain at failure is $\varepsilon_{f}=0.12778$.

The example has been computed with the finite element code ABAQUS [21] by using shell finite element S4R with 5 integration points through the thickness. Only one half of the considered geometry was discretized, see Figs. 6 and 7 . The symmetry conditions $u_{z}=\varphi_{x}=\varphi_{y}=0$ were used in the symmetry plane. The mesh consists of equal squared elements. The free-end cross-section of the model was made rigid by coupling the degrees of freedom of that cross-section.

The plasticity models with strain softening are mesh-dependent. In order to minimize that effect, we have adjusted the post peak uniaxial stress-strain relation to fit the mesh size, as suggested in [4]. According to [4] the linear softening modulus is computed as

$$
K_{s}^{l_{e}}=-\frac{l_{e} \sigma_{u}^{2}}{2 g_{s}}
$$

where $l_{e}$ is a characteristic dimension of the element (in the present case $l_{e}=$ $5 \mathrm{~cm}$ is the side-length of the elements) and $g_{s}$ is the plastic work density (plastic work per unit volume) in the softening regime of the shell material that corresponds to the gray-colored area in Figure 5 (in the present case $g_{s}=$ $0.5 \mathrm{kN} / \mathrm{cm}^{2}$ ). The strain at failure, adjusted to the mesh, is then

$$
\varepsilon_{f}^{l_{e}}=\varepsilon_{u}+\frac{\sigma_{u}}{\frac{E K_{s}^{l_{e}}}{E+K_{s}^{l_{e}}}}=0.10727
$$

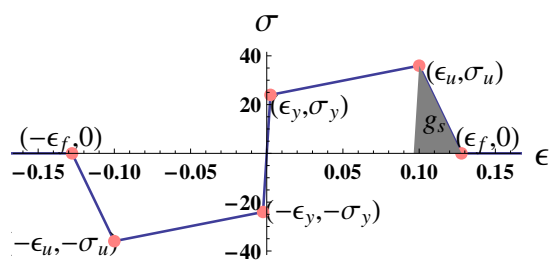

Figure 5: Uniaxial stress-strain curve

The load was applied in two steps. In the first step we applied a desired level of axial force $N$ at the mid-point $M P$ of the rigid cross-section, see Fig. 6 . In the second step we applied bending moment $M$ at the point $M P$ and 


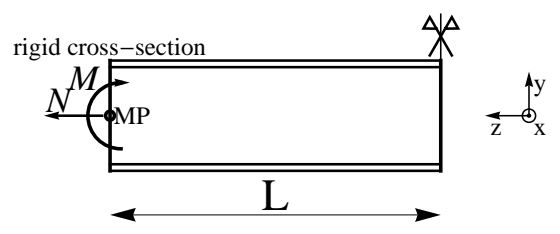

Figure 6: Boundary conditions for the shell model analysis

performed nonlinear analysis with the path-following method. Several analyses were carried out with different values of the axial force (from $N=-0.3 N_{y}$ to $N=0.3 N_{y}$, where $N_{y}=A \sigma_{y}$ ). For each case we monitored the response until the bending resistance dropped to zero (or the analysis ran into convergence problems).

The results of analyses are presented in Figs. 7 to 9. Final deformed configuration of the shell model and distribution of the equivalent plastic strain are presented in Fig. 7 for pure bending case $(N=0)$. We can see that the considered part of the frame member failed by localized buckling of the bottom flange. We also note strong localized yielding of the flange which is concentrated in the neighborhood of the web. Such failure mode was observed also for all other cases. In Figure 8, we show the corresponding moment-rotation

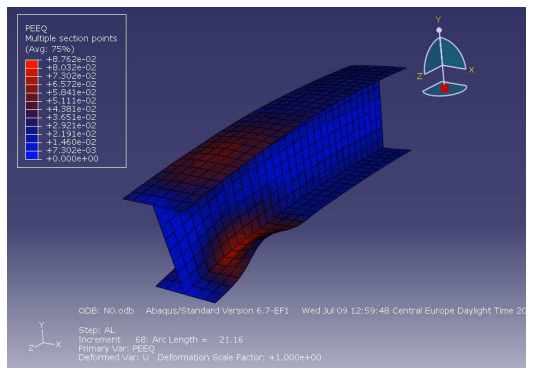

Figure 7: Failure mode of the representative part of the frame member as computed by the shell model

curves. We can see that the level of axial force has a significant influence on the peak bending resistance and on the overall response. In Figure 9 we present the plastic work versus rotation curves. Here, the value of the axial force does not influence much the shape of the curve. The relation between rotation and plastic work is almost linear. In figures 8 and 9 , we marked the points with the maximum bending moment. We assume that those points separate hardening regime from softening regime.

The obtained results by the shell model are now used for evaluation of the beam model material parameters. In Table 1 we summarized the following shell model results: the maximum bending moment $M_{u}^{r e f}$, the plastic work in hardening regime $E^{\bar{W}^{p, r e f}}$, and the plastic work in softening regime $E^{\overline{\bar{W}}^{p, r e f}}$ for different values of axial force $N$. We can see that $M_{u}^{r e f}$ decreases if $N$ is compressive, whereas the tensile axial force has only slight effect on $M_{u}^{r e f}$.

By using Table 1, we determined a bilinear approximation function for $M_{u}^{r e f}$ 


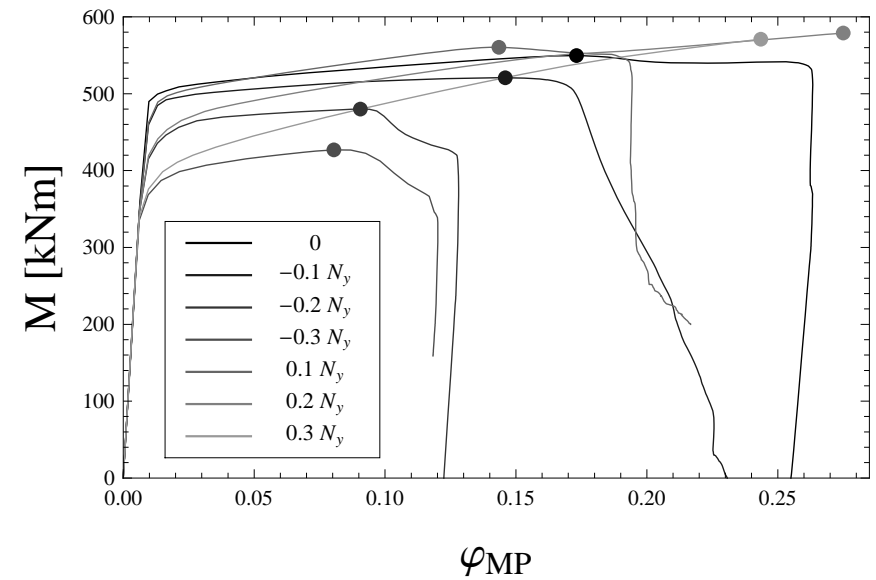

Figure 8: Bending moment versus rotation curves for the end cross-section

\begin{tabular}{|c|c|c|c|}
\hline$N / N_{y}$ & $M_{u}^{\text {ref. }}[\mathrm{kNm}]$ & $E^{\bar{W}^{p, \text { ref. }}}[\mathrm{kJ}]$ & $E^{\overline{\bar{W}}^{\text {pref. }}}[\mathrm{kJ}]$ \\
\hline 0 & 550 & 86 & 53 \\
\hline-0.1 & 521 & 73 & 45 \\
\hline-0.2 & 480 & 46 & 29 \\
\hline-0.3 & 427 & 43 & 27 \\
\hline 0.1 & 560 & 72 & 32 \\
\hline 0.2 & 579 & 149 & 0 \\
\hline 0.3 & 571 & 142 & 0 \\
\hline
\end{tabular}

Table 1: Summary of results of the shell model analyses

as

$$
\widetilde{M}_{u}^{r e f}(N)=\left\{\begin{array}{cl}
M_{u}^{r e f, 0}\left(1.03+0.85 \frac{N}{N_{y}}\right) & \text { if } N<-0.035 N_{y} \\
M_{u}^{r e f, 0} & \text { if } N \geq-0.035 N_{y}
\end{array},\right.
$$

where $M_{u}^{r e f, 0}=M_{u}^{r e f}(N=0)$. We assume that $\widetilde{M}_{u}^{r e f}(N)$ can be used to evaluate the ultimate bending moment of the beam model $M_{u}$, i.e.

$$
M_{u}(N)=\widetilde{M}_{u}^{r e f}(N),
$$

see Fig. 10. The values for the ultimate resistance obtained with the shell analyses are marked with dots in Fig. 10.

The beam model hardening modulus $K_{h}$ can be evaluated point-wise by using (59), (54), (87) and third column of Table 1 . We get $K_{h}$ ranging from $6.26 \cdot 10^{6} \mathrm{kN} / \mathrm{cm}^{2}$ to $1.35 \cdot 10^{7} \mathrm{kN} / \mathrm{cm}^{2}$ with an average value of $1.06 \cdot 10^{7} \mathrm{kN} / \mathrm{cm}^{2}$. Although one could easily find a higher-order function that fits these results, we assume for simplicity that the axial force has no influence on the hardening modulus and adopt

$$
K_{h}(N)=1.06 \cdot 10^{7} \mathrm{kN} / \mathrm{cm}^{2} .
$$

The beam model softening modulus $K_{s}$ can be evaluated point-wise by using (62), (87) and the last column of Table 1 . The gray-colored fields in Table 1 


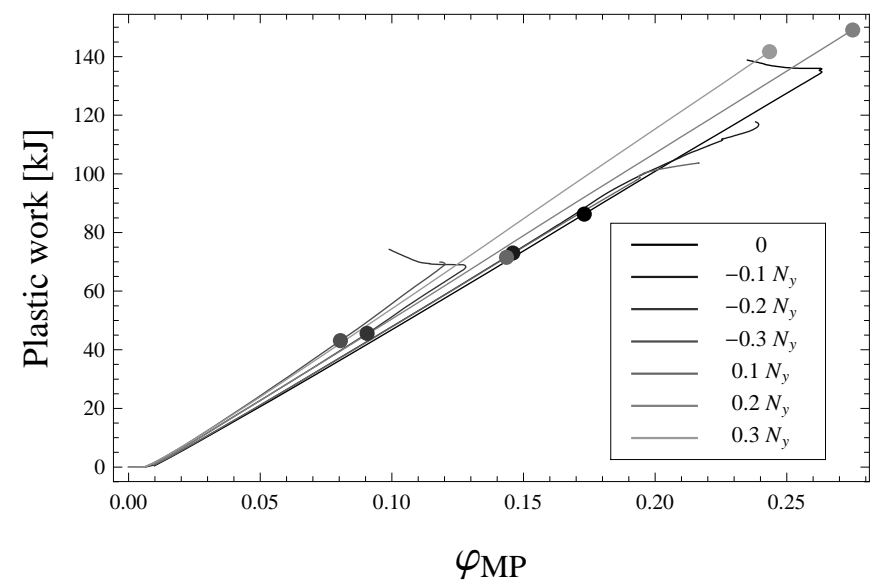

Figure 9: Plastic work versus end cross-section rotation curves

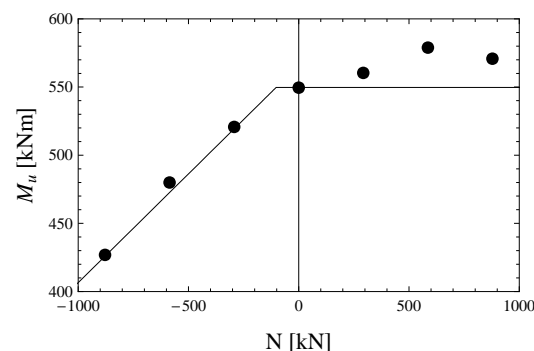

Figure 10: Approximation of the ultimate bending moment of the cross-section

present unreliable results for $E^{\overline{\bar{W}}^{p, r e f}}$, since for those cases the shell analysis did not converge. Softening modulus for the first three analyses ranges from $-2.85 \cdot 10^{5} \mathrm{kN} / \mathrm{cm}^{2}$ to $-3.97 \cdot 10^{5} \mathrm{kN} / \mathrm{cm}^{2}$. We assume that the axial force has no influence on softening modulus and adopt the average value

$$
K_{s}(N)=-3.28 \cdot 10^{5} \mathrm{kN} / \mathrm{cm}^{2} .
$$

In Table 2 we make a point-wise comparison between the shell analysis results $M_{u}^{r e f}, E^{\bar{W}^{p, r e f}}$ and $E^{\overline{\bar{W}}^{p, r e f}}$ and the corresponding beam model results $M_{u}, E^{\bar{W}^{p}}$ and $E^{\overline{\bar{W}}^{p}}$, computed by using approximations (87), (88), (89) and expressions (57), (60) and (52). We can see that the error in ultimate bending moment is small, while the error in dissipated plastic work can be quite large.

\subsection{Push-over of a symmetric frame}

In this example we present a push-over analysis of a symmetric frame. The geometry is given in Fig. 11, where $L_{B}=500$ and $H_{C}=250 \mathrm{~cm}$. The material and cross-section properties of all frame members are equal. They are the same 


\begin{tabular}{|c|c|c|c|c|c|c|}
\hline$N / N_{y}$ & $M_{u}[\mathrm{kNm}]$ & $\left|\frac{M_{u}-M_{u}^{\text {ref. }}}{M_{u}^{\text {ref. }}}\right|[\%]$ & $E^{\bar{W}^{p}}[\mathrm{~kJ}]$ & $\left|\frac{E^{W^{p}}-E^{W^{p, r e f .}}}{E^{W^{p, r e f .}}}\right|[\%]$ & $E^{\overline{\bar{W}}^{p}}[\mathrm{~kJ}]$ & $\left|\frac{E^{\bar{W}^{p}}-E^{\overline{\bar{W}}^{p, \text { ref. }}}}{E^{\bar{W}^{p, r e f .}}}\right|[\%]$ \\
\hline 0 & 550 & 0.00 & 50 & 42 & 46 & 12 \\
\hline-0.1 & 519 & 0.26 & 59 & 20 & 41 & 8 \\
\hline-0.2 & 473 & 1.54 & 54 & 17 & 34 & 19 \\
\hline-0.3 & 426 & 0.22 & 48 & 12 & 28 & $?$ \\
\hline 0.1 & 550 & 1.94 & 81 & 14 & 46 & $?$ \\
\hline 0.2 & 550 & 5.07 & 109 & 27 & 46 & $?$ \\
\hline 0.3 & 550 & 3.72 & 134 & 6 & 46 & $?$ \\
\hline
\end{tabular}

Table 2: Comparison between approximations and shell analyses results

as those presented in Section 5.1. The vertical load is constant and equals $q_{v}=$ $0.05 \mathrm{kN} / \mathrm{cm}$. The lateral loading is presented in Figure 11, where $F_{0}=1 \mathrm{kN}$ is a concentrated force and $\lambda$ is load multiplier. The mesh consists of eight beam finite elements per each frame member.

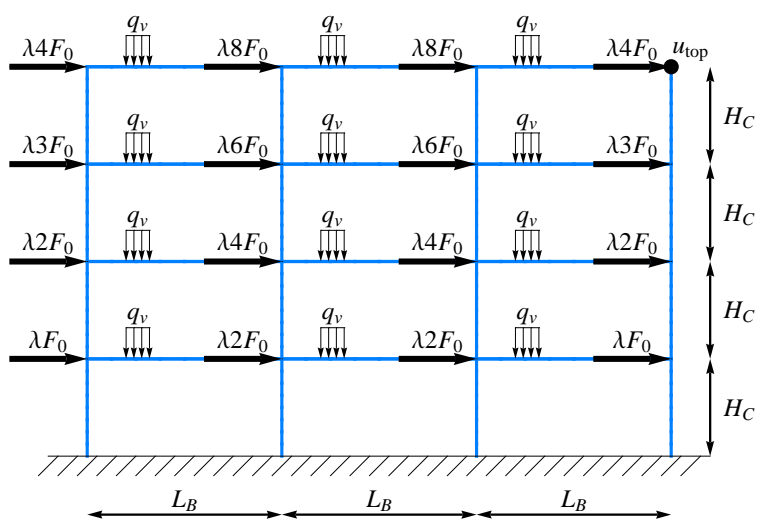

Figure 11: Symmetric frame: geometry and loading

We performed two analyses, one by using the geometrically linear and the other by using the geometrically nonlinear beam finite elements. The results are presented in Figures 12 and 13, where $u_{t o p}$ is horizontal displacement of the top right corner of the frame. In the left part of the Fig. 12 we present the total lateral load versus $u_{\text {top }}$ curves. The points on those curves mark configurations where the softening hinge was activated in one of the elements of the mesh. We can see that, even though some parts of the frame are failing, the total resistance of the structure is still growing until the maximum load is reached at $1527.3 \mathrm{kN}$ for geometrically linear case, and at $1522.3 \mathrm{kN}$ for geometrically nonlinear case.

In the right part of Fig. 12 we present the plastic work versus $u_{t o p}$ displacement curves. The results of the geometrically linear and the geometrically nonlinear elements are completely the same. At the beginning of the analysis there is no energy dissipation since the material response is elastic. The nondissipative period is followed by a short period with dissipation due to material hardening only, which ends with the first activation of softening plastic hinge in one of the beam finite elements. For a while we have a combined hardening and softening energy dissipation, which is finally followed by a period when the structure is dissipating energy only due to softening. On the left part of Fig. 13 we present the final deformed configuration of the frame. In the right part of 

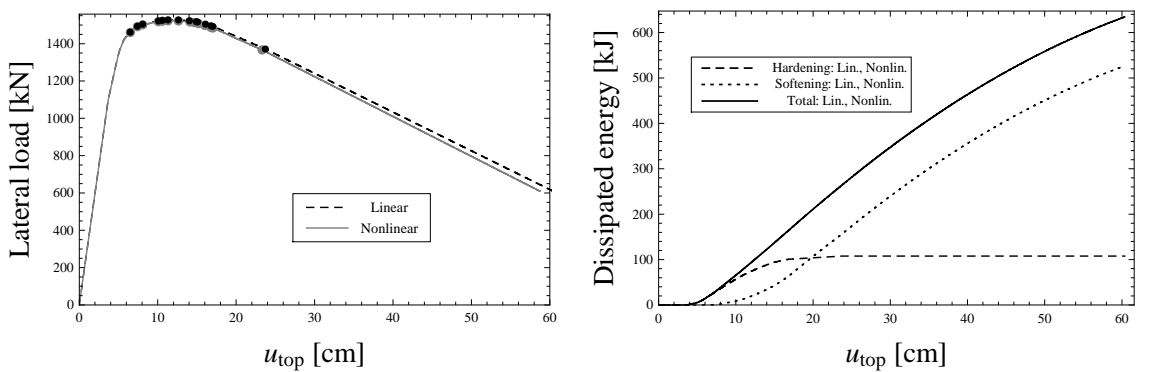

Figure 12: Load versus displacement and dissipated energy versus displacement curves

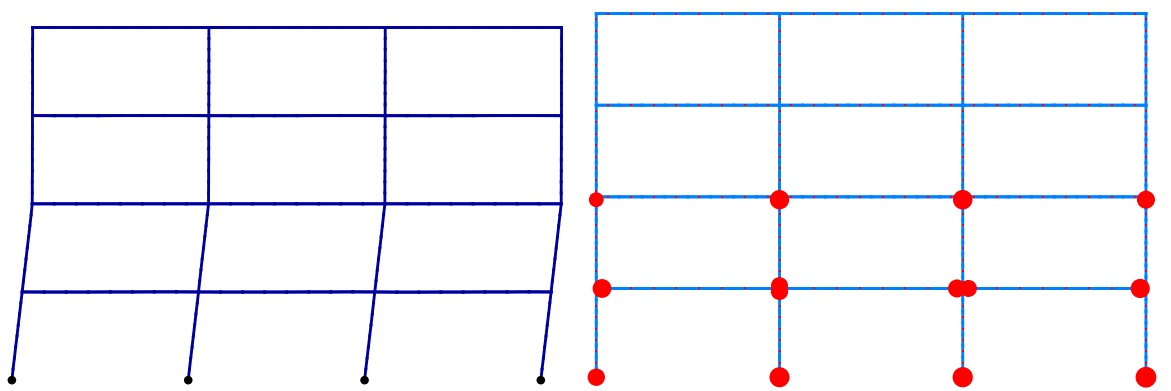

Figure 13: Deformed shape and locations of softening plastic hinges at $u_{t o p} \approx 60$ $\mathrm{cm}$

Fig. 13 we present locations where the softening plastic hinges appeared during the analysis.

\subsection{Push-over of an asymmetric frame}

In this example we analyze an asymmetric frame presented in Fig. 14, where $L_{B 1}=600 \mathrm{~cm}, L_{B 2}=500 \mathrm{~cm}, L_{B 3}=400 \mathrm{~cm}$ and $H_{C}=250 \mathrm{~cm}$. All the other geometrical, material and loading parameters are the same as in the previous example.

The results are presented in Figs. 15 to 16. The total lateral load versus $u_{t o p}$ curves, where $u_{t o p}$ is horizontal displacement at the top-left corner of the frame, are presented on the left part of Fig. 15. The results of geometrically linear and geometrically nonlinear analyses are nearly the same before the ultimate resistance is reached at $1581.8 \mathrm{kN}$ for geometrically linear case and at 1578.4 $\mathrm{kN}$ for geometrically nonlinear case. After that point the difference between those two analyses is bigger. The final computed equilibrium configuration for the geometrically nonlinear case is at $u_{t o p}=26.52 \mathrm{~cm}$. In the next load step one additional softening plastic hinge is activated, which results in the global failure mechanism. Since our path-following algorithm is only governed by the the increase of $u_{t o p}$, we are unable to capture the remaining part of the loaddisplacement curve.

In the right part of Fig. 15 we present the dissipated energy versus $u_{t o p}$ 


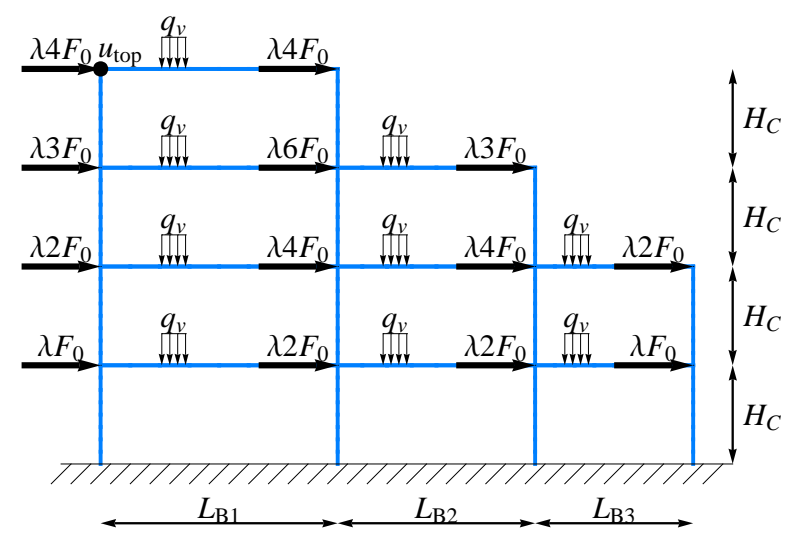

Figure 14: Asymmetric frame: geometry and loading
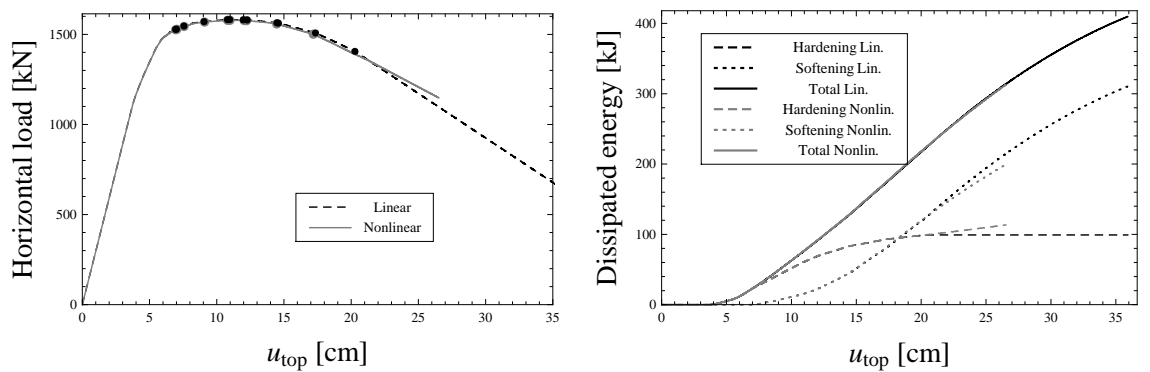

Figure 15: Load versus displacement and dissipated energy versus displacement curves

curves. The shapes of the curves are very similar to those from the symmetric frame case. Namely, first there is the elastic non-dissipative phase, followed by the pure hardening dissipation phase, followed by the combined hardening and softening dissipation phase and finally the pure softening dissipation phase. In the geometrically nonlinear case we do not have the final pure softening dissipation phase due to activation of global failure mechanism. On the left part of Fig. 16 we present the deformed configuration of the frame at $u_{t o p}=26.52 \mathrm{~cm}$. Locations, where softening plastic hinges were activated at $u_{\text {top }} \approx 26.52 \mathrm{~cm}$, are presented in the middle part of Fig. 16 for the geometrically linear case and in the right part of the same figure for the geometrically nonlinear case.

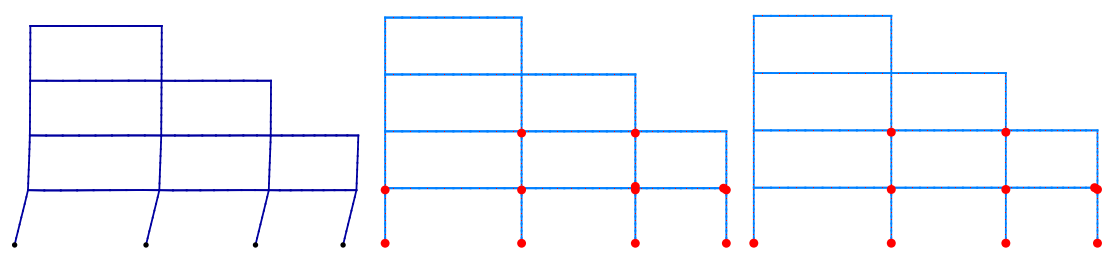

Figure 16: Deformed shape and locations of softening plastic hinges 


\subsection{Bending of beam under constant axial force}

In this example we compare results of the beam model with results obtained by using the shell finite element model from ABAQUS. For the comparison we choose the problem of the bending of the beam of length $L^{\text {ref }}$ under a constant axial force, presented in Section 5.1. For that reason, the geometric and material properties are the same as those in the Section 5.1. The beam model analyses are performed with two sets of material parameters, where the first set (SET1) is given by (87) to (89). In the second set (SET2) we replace the expression (87) with

$$
M_{u}^{*}=M_{u}^{(87)}-N\left|\Delta u_{y}\right|,
$$

where $M_{u}$ is the maximum concentrated moment applied at the end cross-section (point $M P$, see Fig. 6), $N$ is the applied axial force (positive when producing tension) and $\Delta u_{y}$ is the relative displacement in the $y$ direction between the point $M P$ and the position of the local failure. The difference between the applied concentrated moment at the point $M P$ (see Fig. 6) and $M_{u}^{*}$ thus arises due to large displacements correction. When the yielding and local buckling of the beam are significant and the displacements in the $y$ direction are no longer negligible, the contribution of the axial force $N$ to the bending moment must be taken into account. In this particular case we have $N=-0.1 N y$, $M_{u}=521 \mathrm{kNm}$ and $\Delta u_{y}=0.15 \mathrm{~m}$, which leads to $M_{u}^{*}=565 \mathrm{kNm}$.

Five beam finite elements are used to model one-half of the beam under consideration, since the symmetry is taken into account. The symmetry conditions at the symmetry plane are $u=w=w^{\prime}=0$. The load was applied in two steps. In the first step the beam was loaded with compressive axial force $N=-0.1 N_{y}$. In the second step the moment $M$ was applied at the free-end of the beam. In order to ensure the proper activation of softening in the geometrically linear analyses the ultimate bending moment of the finite element near the symmetry plane was slightly weakened.
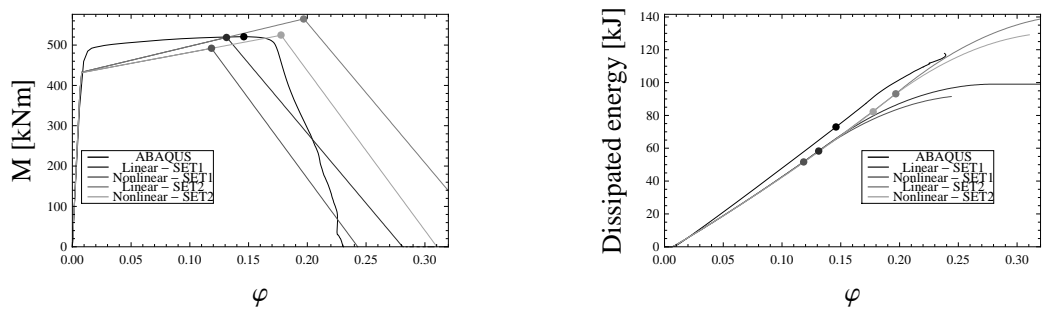

Figure 17: Comparison of results for the bending of the beam under compression axial force

In Fig. 17 the results for geometrically linear and geometrically nonlinear cases are compared with the results of the shell model from ABAQUS. On the left part of Fig. 17 we present curves relating applied moment to free-end rotation. The ultimate bending moments of the shell model, the geometrically linear SET1 beam model and the geometrically nonlinear SET2 beam model are very close, whereas the geometrically linear SET2 beam model gives slightly 
bigger and the geometrically nonlinear SET1 beam model gives slightly lower value for ultimate bending moment.

On the right part of Figure 17 we present the plastic work versus rotation of the end cross-section curves. There is hardly any difference between the beam model results when $\varphi$ is smaller than 0.15 . After that point the difference becomes bigger. The prediction of the beam model for plastic work in hardening regime is in the case of geometrically linear analysis with SET1 material parameters $80 \%$ of the shell model prediction, and the prediction for plastic work in softening regime is $92 \%$ of the shell model prediction. This is in agreement with the results of Table 2. The predictions for plastic work of other beam analyses give bigger differences compared to the shell model. We note that one could get better agreements in plastic work by using better approximations for hardening and softening moduli in place of simplifications (88) and (89).

\subsection{Collapse of a simple frame}

In this example we compare results of the nonlinear beam model with the results of the shell model. We consider a simple frame presented in Fig. 18. The geometry of the beam model (middle axes of the beam model correspond to the middle axes of the shell model) is presented on the left part of Fig. 18. The geometry and the finite element mesh of the shell model is presented on the right side of Fig. 18. The cross-section and the material properties of the shell model are the same as those in the section 5.1. In the shell model we made connections between the beam and the columns rigid by coupling the degrees of freedom of the corresponding end cross-sections. The beam model mesh consists of eight finite elements per each frame member. Two different sets of material parameters are used for the beam model analysis. The first set (SET1) is given by (54) and (87) to (89) and the second set (SET2) by

$$
M_{y}=1.2 M_{y}^{(54)}, \quad M_{u}=1.2 M_{u}^{(87)}, \quad K_{h}=0.6 K_{h}^{(88)}, \quad K_{s}=K_{s}^{(89)} .
$$

Support conditions are $\left(u=w=w^{\prime}=0\right.$ for the beam model and $u_{x}=u_{y}=$ $u_{z}=\varphi_{x}=\varphi_{y}=\varphi_{z}=0$ for the shell model).
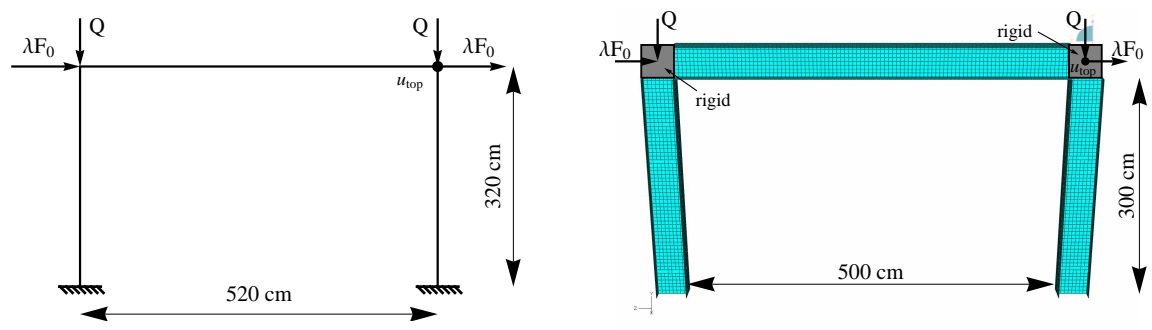

Figure 18: Simple frame: the beam and the shell model

The load applied to the frame is presented in Fig. 18. The vertical load is constant and equal to $Q=500 \mathrm{kN}$, while the horizontal load multiplier $\lambda$ $\left(F_{0}=1 \mathrm{kN}\right)$ is controlled with the path-following method.

The results are presented in Figs. 19 to 21. The total lateral load versus $u_{t o p}$ curves $\left(u_{t o p}\right.$ is horizontal displacement of the top-right corner of the frame) 
are presented on the left part of Fig. 19. The dissipated energy versus $u_{t o p}$ curves are presented on the right part of Fig. 19. On the left part of Fig. 20 we present the equivalent plastic deformation on the deformed configuration of the shell model. The deformed configuration of the beam model, along with positions where the softening response was activated, is presented on the right part of Fig. 20. Note, that in both models the localized failure appears at the ends of the columns. In Fig. 21 we present the internal forces at the right support of the shell model. Note that the axial force is not constant at the beginning of the loading, but once the response becomes nonlinear it hardly changes.

The lateral load versus $u_{\text {top }}$ curve (left part of Fig. 19) of the beam model with SET1 material parameters has a similar shape as the shell model curve, but the prediction of the maximum resistance of the beam model is around $84 \%$ of the shell model's resistance. We have a similar situation as in the previous example, where the resistance of the cross-section was greater than the one obtained by analysis in Section 5.1. On the bottom-right part of Fig. 21 one can see that the axial force that corresponds to the maximum bending moment $M_{\max }=523 \mathrm{kNm}$ is around $N\left(M_{\max }\right)=-720 \mathrm{kN} \approx-0.25 N_{y}$. If we compare $M_{\max }$ to $M_{u}(-0.2 N y)=473 \mathrm{kNm}$ from Table 2 , we can see that we have more than 10\% bigger bending resistance. One must also consider that plasticity (hardening and softening) in the beam model is triggered at positions where we have rigid connections in the shell model, which also decreases the resistance of the beam model compared to the shell model.

The dissipated energy versus $u_{t o p}$ curve (right part of Fig. 19) of the beam model with SET1 material parameters has a similar shape to the shell model's curve. The prediction of the SET1 beam model for the value of the dissipated energy that corresponds to $u_{t o p}=20 \mathrm{~cm}$ is around $71 \%$ of the shell model prediction.

These results are significantly improved, and we obtain much better fit to the shell model, when SET2 beam parameters are used; see Fig. 19. We recall that the latter accounts for the large displacement correction of ultimate resistance.
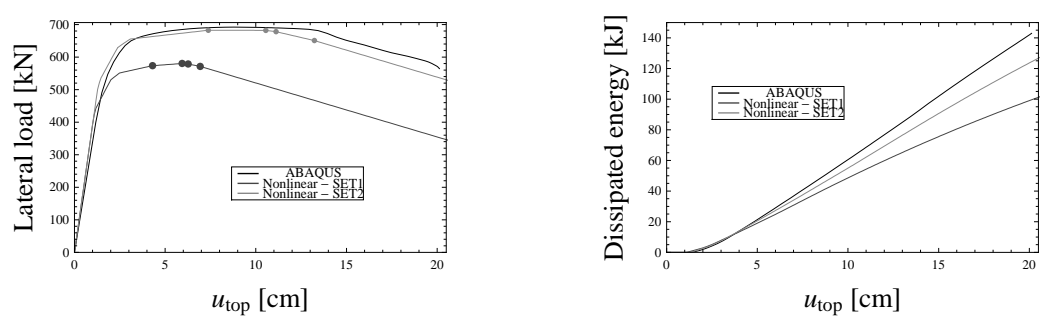

Figure 19: Comparison of results for simple frame example

\subsection{Darvall-Mendis frame}

We consider the clamped portal frame under vertical loading first studied by Darvall and Mendis [22] and later examined by Armero and Ehrlich [7] and 


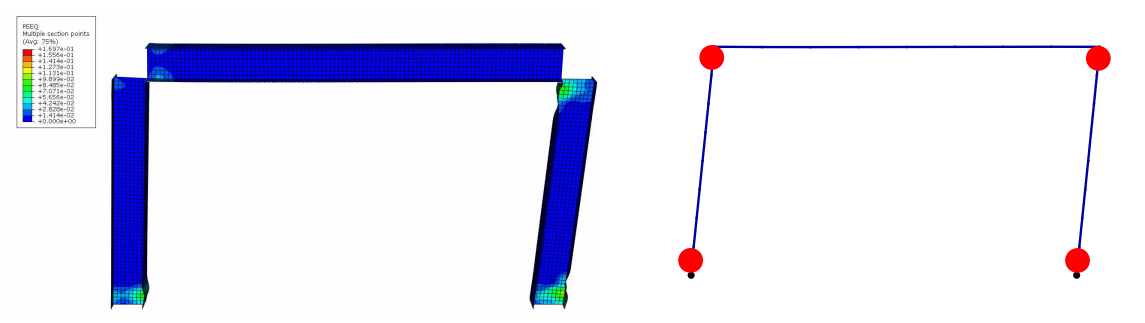

Figure 20: Deformed shapes of the simple frame
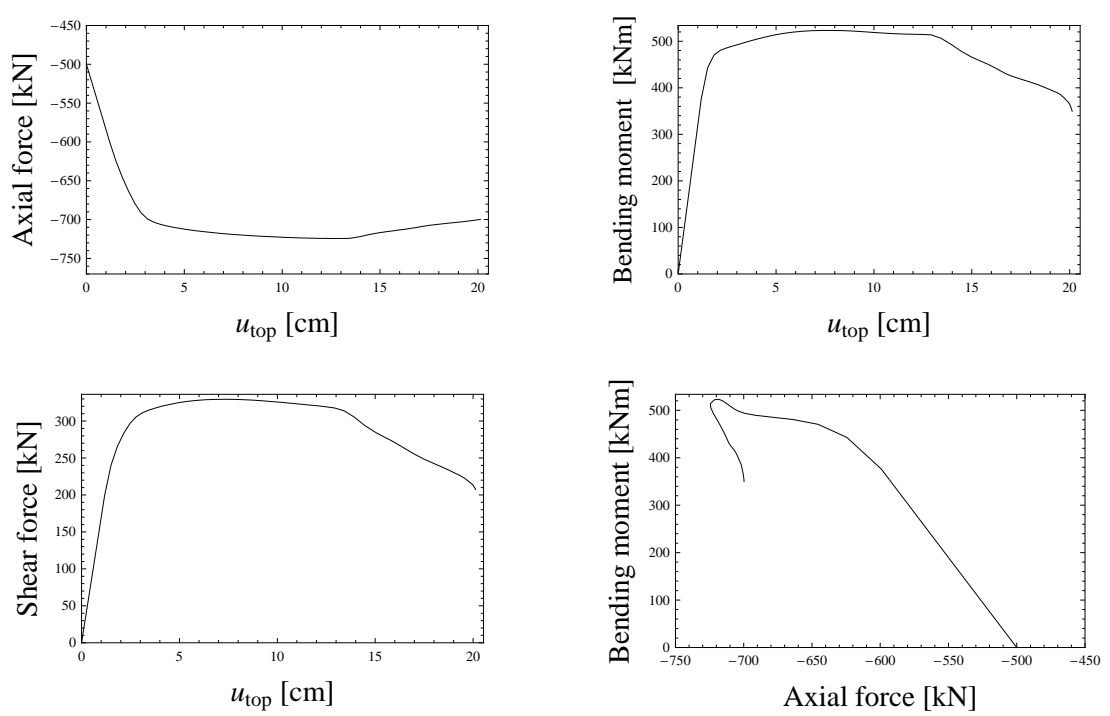

Figure 21: Internal forces at the right support of the shell model

Wackerfuss [11]. The geometry of the frame is presented in Figure 22. The length of the columns and the beam is $L=3.048 \mathrm{~m}$, cross-section area of all the members is $A=0.103 \mathrm{~m}^{2}$ and their moment of inertia is $I=0.001$ $\mathrm{m}^{4}$. The elastic material response is defined by the Young's modulus $E=$ $2.068 \times 10^{7} \mathrm{kN} / \mathrm{m}^{2}$. The inelastic response is defined by the ultimate bending moment $M_{u, C}=158.18 \mathrm{kNm}$ for the columns, the ultimate bending moment $M_{u, B}=169.48 \mathrm{kNm}$ for the beam, and the softening modulus $K_{s}=\frac{a E I}{10 L}$, where the values $a=0,-0.04,-0.06,-0.0718$ are considered. Note, that in this example the inelastic response does not include any material hardening. We also consider that the axial force has no influence on the ultimate bending resistance of the frame members. The mesh consists of eight geometrically linear beam finite elements, see Figure 22. The frame is loaded with a vertical load $\lambda F_{0}$ $\left(F_{0}=1 \mathrm{kN}\right)$ applied at the node 5 , see Figure 22 . In the numerical simulations we control the load multiplier $\lambda$ and the vertical displacement $u_{v}$ at the node 5 by the path-following method.

The vertical load versus vertical deflection curves are presented in Figure 23, where the points on the curves mark configurations where the softening plastic 


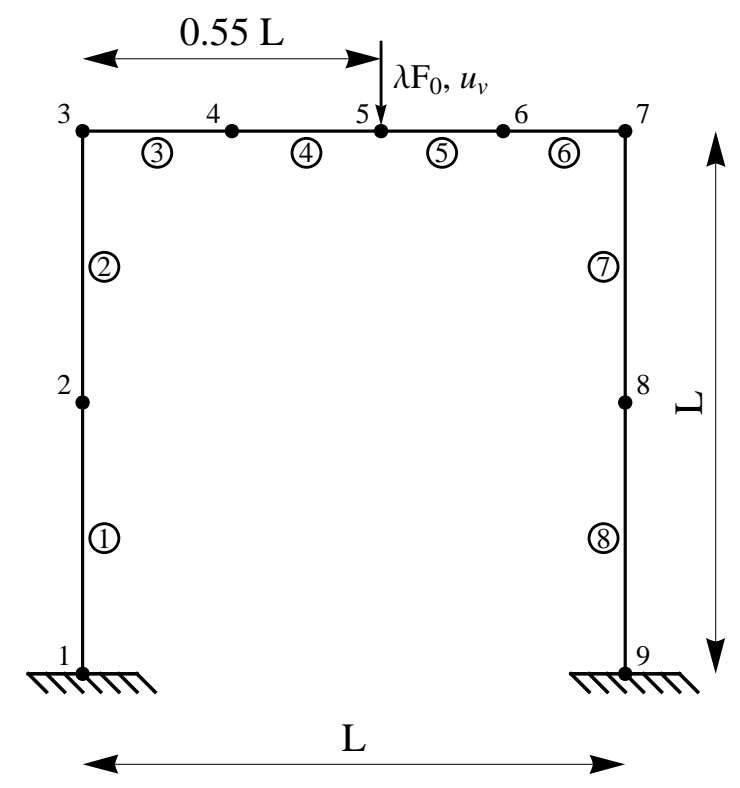

Figure 22: Geometry and loading of the portal frame.

hinge was activated in one of the elements of the mesh. In all cases hinges form at the same locations. The first hinge forms in elements 4 and 5 at node 5 , the second hinge forms in element 7 at node 7 and the third hinge forms in element 2 at node 3 . This example shows the significant influence of the softening modulus on the ultimate load of the structure. The ultimate load in the case of perfectly plastic response of the hinges $(a=0)$ is $434 \mathrm{kN}$ when the third hinge forms. In cases $a=-0.04$ and $a=-0.06$ the structure fails when the second hinge forms when the vertical load reaches $383 \mathrm{kN}$ and $350 \mathrm{kN}$, respectively. In the case of $a=-0.0718$ the structure fails when the first hinge forms at $336 \mathrm{kN}$. In Table 3 we compare our results with the results obtained by Darvall and Mendis [22], Armero and Ehrlich [7] and Wackerfuss [11].

\begin{tabular}{|cc|cc|cc|cc|cc|}
\multicolumn{1}{c}{} & Darvall and Mendis $[22]$ & \multicolumn{2}{c|}{ Armero and Ehrlich $[7]$} & \multicolumn{2}{c|}{ Wackerfuß $[11]$} & \multicolumn{2}{c|}{ Present } \\
\hline \multicolumn{2}{|c|}{ Hinge } & $u_{v}[\mathrm{~cm}]$ & $\lambda F_{0}[\mathrm{kN}]$ & $u_{v}[\mathrm{~cm}]$ & $\lambda F_{0}[\mathrm{kN}]$ & $u_{v}[\mathrm{~cm}]$ & $\lambda F_{0}[\mathrm{kN}]$ & $u_{v}[\mathrm{~cm}]$ & $\lambda F_{0}[\mathrm{kN}]$ \\
\hline 0 & 1 & 0.50 & 336 & 0.50 & 337 & 0.53 & 342 & 0.50 & 336 \\
& 2 & 1.14 & 427 & 1.14 & 428 & 1.13 & 435 & 1.13 & 427 \\
& 3 & 1.34 & 433 & 1.34 & 434 & 1.33 & 440 & 1.34 & 434 \\
\hline-0.04 & 1 & 0.50 & 336 & 0.50 & 337 & 0.53 & 349 & 0.50 & 336 \\
& 2 & 1.14 & 387 & 1.18 & 388 & 1.16 & 401 & 1.19 & 383 \\
\hline-0.06 & 1 & 0.50 & 336 & 0.50 & 337 & 0.52 & 348 & 0.50 & 336 \\
& 2 & 1.19 & 336 & 1.29 & 337 & 1.23 & 349 & 1.23 & 350 \\
\hline-0.0718 & 1 & 0.50 & 336 & 0.50 & 337 & & & 0.50 & 336 \\
\hline
\end{tabular}

Table 3: Comparison of the presented formulation with the literature

\section{Conclusion}

The presented multi-scale model for computing the limit load of planar metal frames under the push-over and the full collapse analysis combines the best of both worlds: on one side the effectiveness and robustness of the macro-scale 


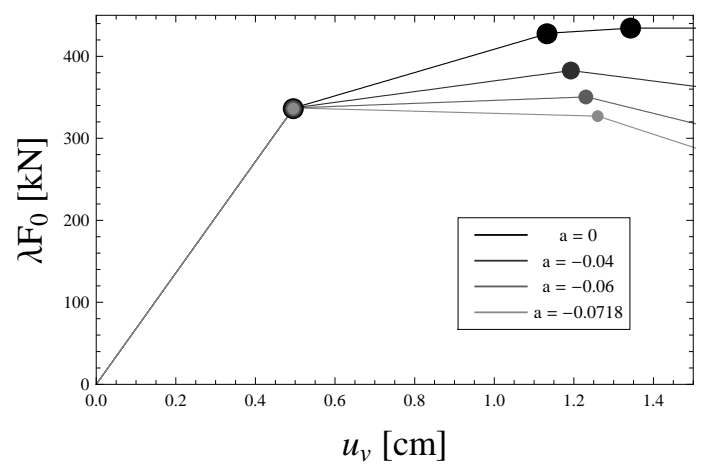

Figure 23: Vertical load versus vertical deflection curves

beam model for the entire structure, and on another side, a refined representation of localized instability effects (both geometric and material) by meso-scale effects based upon the geometrically nonlinear elastoplastic shell formulation. The latter is captured and stored within the macro-scale beam model in the manner which is compatible with enhanced beam kinematics with embedded discontinuity. The most appropriate choice of the meso-scale shell model can be further guided by the error-controlled adaptive finite element method for shell structures (by using model error estimation, see e.g. Bohinc et al. [23]), which could automatically find the most appropriate model for representing a particular local phenomena under consideration.

The multiscale procedure proposed in this paper belongs to the class of weak coupling methods, where we carry out the sequential computations. The results of the shell model computations, accounting for material and geometric localized instability, are stored to be used within the beam model softening response. As presented by numerical simulations, performance of the proposed multi-scale computational approach is very satisfying. One of its main features is that detection and development of the softening plastic hinges in the frame is fully automatic, and spreads gradually in accordance with stress redistribution in the course of the nonlinear analysis. This is in contrast with many standard computational approaches to the limit load, under the push-over and the full collapse analysis of frames, which rely on predefined locations of plastic hinges and the corresponding inelastic deformations.

\section{References}

[1] P. Fajfar, M. Dolšek, D. Marušić, A. Stratan, Pre- and post-test mathematical modelling of a plan-asymmetric reinforced concrete frame building, Earthquake Engng. and Struct. Dyn., 35, 1359-1379, 2006.

[2] E.L. Wilson, Three dimensional static and dynamic analysis of structures, CSi, 2002.

[3] G.H. Powell, Nonlinear structural analysis by computer code INSA, UC Berkeley reports SEMM 86-15, 1986. 
[4] A. Ibrahimbegovic, Nonlinear Solid Mechanics: Theoretical Formulations and Finite Element Solution Methods, Springer, 2009.

[5] M. Jirasek, Analytical and numerical solutions for frames with softening hinges, ASCE J. Eng. Mech. Din., 123, 8-14, 1997.

[6] D. Ehrlich, F. Armero, Finite element methods for the analysis of softening plastic hinges in beams and frames, Comput. Mech., 35, 237-264, 2005.

[7] F. Armero, D. Ehrlich, Numerical modeling of softening hinges in thin Euler-Bernoulli beams, Computers and Structures, 84, 641-656, 2006.

[8] F. Armero, D. Ehrlich, An analysis of strain localization and wave propagation in plastic models of beams at failure, Comput. Meth. Appl. Mech. Eng., 193, 3129-3171, 2004.

[9] A. Ibrahimbegovic, S. Melnyk, Embedded discontinuity finite element method for modelling of localized failure in heterogeneous materials with structured mesh: an alternative to extended finite element method, Comput. Mech., 40, 149-155, 2007.

[10] A. Ibrahimbegovic, D. Brancherie, Combined hardening and softening constitutive model of plasticity: precursor to shear slip line failure, Comput. Mech., 31, 88-100, 2003.

[11] J. Wackerfuss, Efficient finite element formulation for the analysis of localized failure in beam structures, Int. J. Numer. Meth. Engng., 73, 1217-1250, 2008.

[12] A. Kucerova, D. Brancherie, A. Ibrahimbegovic, J. Zeman, Z.Bittnar, Novel anisotropic continuum-discrete damage model capable of representing localized failure of massive structures. Part II: identification from tests under heterogeneous stress field, Engineering Computations, 26, 128-144, 2009.

[13] B. Brank, D. Perić, F.B. Damjanić, On large deformations of thin elastoplastic shells: Implementation of a finite rotation model for quadrilateral shell element, Int. J. Numer. Meth. Engng., 40, 689-726, 1997.

[14] B. Brank, Nonlinear shell models with seven kinematic parameters, Comput. Meth. Appl. Mech. Eng., 194, 2336-2362, 2005.

[15] J.N. Reddy, An introduction to nonlinear finite element analysis, Oxford University Press, 2004.

[16] A. Ibrahimbegovic, F. Gharzeddine, L. Chorfi, Classical plasticity and viscoplasticity models reformulated: Theoretical basis and numerical implementation, Int. J. Numer. Meth. Engng., 42, 1499-1535, 1998.

[17] J. Lubliner, Plasticity theory, Macmillian, 1990.

[18] J.C. Simo, T.J.R. Hughes, Computational inelasticity, Springer, 1998.

[19] J. Dujc, B. Brank, On stress resultant plasticity and viscoplasticity for metal plates, Finite Elements in Analysis and Design, 44, 174-185, 2008. 
[20] J. Korelc, AceGen, AceFem, available at http://www.fgg.uni-lj.si/Symech.

[21] Hibbit, Karlsson, Sorensen, Abaqus Manuals.

[22] P.L. Darvall, P.A. Mendis, Elastic-plastic-softening analysis of plane frames, Journal of Structural Engineering (ASCE), 1985, 111, 871-888.

[23] U. Bohinc, A. Ibrahimbegovic, B. Brank, Model adaptivity for finite element analysis of thin and thick plates based on equilibrated boundary stress resultants, Engineering Computations, 26, 69-99, 2009. 